\title{
On the Evolution of Syntactic Information Encoded by BERT's Contextualized Representations
}

\author{
Laura Pérez-Mayos ${ }^{1}$, Roberto Carlini ${ }^{1}$, Miguel Ballesteros ${ }^{2}$, Leo Wanner ${ }^{3,1}$ \\ ${ }^{1}$ TALN Research Group, Pompeu Fabra University, Barcelona, Spain \\ 2 Amazon AI \\ ${ }^{3}$ Catalan Institute for Research and Advanced Studies (ICREA), Barcelona, Spain \\ \{laura.perezm|roberto.carlini|leo.wanner\}@upf.edu \\ ballemig@amazon.com
}

\begin{abstract}
The adaptation of pretrained language models to solve supervised tasks has become a baseline in NLP, and many recent works have focused on studying how linguistic information is encoded in the pretrained sentence representations. Among other information, it has been shown that entire syntax trees are implicitly embedded in the geometry of such models. As these models are often fine-tuned, it becomes increasingly important to understand how the encoded knowledge evolves along the fine-tuning. In this paper, we analyze the evolution of the embedded syntax trees along the fine-tuning process of BERT for six different tasks, covering all levels of the linguistic structure. Experimental results show that the encoded syntactic information is forgotten (PoS tagging), reinforced (dependency and constituency parsing) or preserved (semanticsrelated tasks) in different ways along the finetuning process depending on the task.
\end{abstract}

\section{Introduction}

Adapting unsupervised pretrained language models (LMs) to solve supervised tasks has become a widely spread practice in NLP, with models such as ELMo (Peters et al., 2018) and, most notably, BERT (Devlin et al., 2019), achieving state-of-the-art results in many well-known Natural Language Understanding benchmarks like GLUE (Wang et al., 2018) and SQuAD (Rajpurkar et al., 2018). Several studies investigate what the LMs learn, how and where the learned knowledge is represented and what the best methods to improve it are; cf., e.g., (Rogers et al., 2020). There is evidence that, among other information (such as, e.g., PoS, syntactic chunks and roles (Tenney et al., 2019b; Lin et al., 2019; Belinkov et al., 2017), morphology in general (Peters et al., 2018), or sentence length (Adi et al., 2016)) BERT deep models' vector geometry implicitly embeds entire syntax trees
(Hewitt and Manning, 2019). However, rather little is understood about how these representations change when fine-tuned to solve downstream tasks (Peters et al., 2019).

In this work, we aim to understand how syntax trees implicitly embedded in the geometry of deep models evolve along the fine-tuning process of BERT on different supervised tasks, and shed some light on the importance of the syntactic information for those tasks. Intuitively, we expect morpho-syntactic tasks to clearly reinforce the encoded syntactic information, while tasks that are not explicitly syntactic in nature should maintain it in case they benefit from syntax (Kuncoro et al., 2020) and lose it if they do not. In order to cover the three main levels of the linguistic description (morphology, syntax and semantics), we select six different tasks: PoS tagging, constituency parsing, syntactic dependency parsing, semantic role labeling (SRL), question answering (QA) and paraphrase identification. The first three inherently deal with (morpho-)syntactic information while the latter three, which traditionally draw upon the output of syntactic parsing (Carreras and Màrquez, 2005; Björkelund et al., 2010; Strubell et al., 2018; Wang et al., 2019, inter-alia), deal with higher level, semantic information. Almost all of our experiments are on English corpora; one is on multilingual dependency parsing.

\section{Related work}

BERT has become the default baseline in NLP, and consequently, numerous studies analyze its linguistic capabilities in general (Rogers et al., 2020; Henderson, 2020), and its syntactic capabilities in particular (Linzen and Baroni, 2020). Even if syntactic information is distributed across all layers (Durrani et al., 2020), BERT captures most phrase-level information in the lower layers, followed by surface 
features, syntactic features and semantic features in the intermediate and top layers (Jawahar et al., 2019; Tenney et al., 2019a; Hewitt and Manning, 2019). The syntactic structure captured by BERT adheres to that of the Universal Dependencies (Kulmizev et al., 2020); different syntactic and semantic relations are captured by self-attention patterns (Kovaleva et al., 2019; Limisiewicz et al., 2020; Ravishankar et al., 2021), and it has been shown that full dependency trees can be decoded from single attention heads (Ravishankar et al., 2021). BERT performs remarkably well on subject-verb agreement (Goldberg, 2019), and is able to do full parsing relying only on pretraining architectures and no decoding (Vilares et al., 2020), surpassing existing sequence labeling parsers on the Penn Treebank dataset (De Marneffe et al., 2006) and on the end-to-end Universal Dependencies Corpus for English (Silveira et al., 2014). It can generally also distinguish good from bad completions and robustly retrieves noun hypernyms, but shows insensitivity to the contextual impacts of negation (Ettinger, 2020).

Different supervised probing models have been used to test for the presence of a wide range of linguistic phenomena in the BERT model (Conneau et al., 2018; Liu et al., 2019; Tenney et al., 2019b; Voita and Titov, 2020; Elazar et al., 2020). Hewitt and Manning (2019)'s structural probe shows that entire syntax trees are embedded implicitly in BERT's vector geometry. Extending their work, Chi et al. (2020) show that multilingual BERT recovers syntactic tree distances in languages other than English and learns representations of syntactic dependency labels.

Regarding how fine-tuning affects the representations of BERT, Gauthier and Levy (2019) found a significant divergence between the final representations of models fine-tuned on different tasks when using the structural probe of Hewitt and Manning (2019), while Merchant et al. (2020) concluded that fine-tuning is conservative and does not lead to catastrophic forgetting of linguistic phenomena which our experiments do not confirm. However, we find that the encoded syntactic information is forgotten, reinforced or preserved differently along the fine-tuning process depending on the task.

\section{Experimental setup}

We study the evolution of the syntactic structures discovered during pretraining along the fine-tuning of BERT-base (cased) (Devlin et al., 2019) ${ }^{1}$ on six different tasks, drawing upon the structural probe of Hewitt and Manning (2019). ${ }^{2}$ We fine-tune the whole model on each task outlined below for 3 epochs, with a learning rate of $5 e^{-5}$, saving 10 evenly-spaced checkpoints per epoch. The output of the last layer is used as input representation for the classification components of each task. To mitigate the variance in performance induced by weight initialization and training data order (Dodge et al., 2020), we repeat this process 5 times per task with different random seeds and average results.

PoS tagging. We fine-tune BERT with a linear layer on top of the hidden-states output for token classification. ${ }^{3}$ Dataset: Universal Dependencies Corpus for English (UD 2.5 EN EWT Silveira et al. (2014)).

Constituency parsing. Following Vilares et al. (2020), we cast constituency parsing as a sequence labeling problem, and use a single feed-forward layer on top of BERT to directly map word vectors to labels that encode a linearized tree. Dataset: Penn Treebank (Marcus et al., 1993).

Dependency parsing. We fine-tune a Deep Biaffine neural dependency parser (Dozat and Manning, 2016) on three different datasets: i) UD 2.5 English EWT (Silveira et al., 2014); ii) a multilingual benchmark generated by concatenating the UD 2.5 standard data splits for German, English, Spanish, French, Italian, Portuguese, and Swedish (Zeman et al., 2019), with gold PoS tags; iii) PTB SD 3.3.0 (De Marneffe et al., 2006).

Semantic role labeling. Following Shi and Lin (2019), we decompose the task into i) predicate sense disambiguation and argument identification, and ii) classification. Both subtasks are casted as sequence labeling, feeding the contextual representations into a one-hidden-layer MLP for the first, and a one-layer BiLSTM followed by a one-hiddenlayer MLP for the latter. Dataset: OntoNotes corpus (Weischedel et al., 2013).

Question answering. We fine-tune BERT with

\footnotetext{
${ }^{1}$ Our experiments are implemented in PyTorch, using two open-source libraries: the Transformers library (Wolf et al., 2019) and AllenNLP (Gardner et al., 2017). Implementation details, pretrained weights and full hyperparameter values can be found in the libraries documentation.

${ }^{2}$ We use the same experimental setup used by the authors. Source: https://github.com/john-hewitt/ structural-probes

${ }^{3}$ Source: https://github.com/Tarpelite/ UniNLP/blob/master/examples/run_pos.py
} 
a linear layer on top of the hidden-states output to compute span start logits and span end logits. ${ }^{4}$ Dataset: Stanford Question Answering Dataset (SQuAD (Rajpurkar et al., 2018)).

Paraphrase identification. We fine-tune BERT with a linear layer on top of the pooled sentence representation. ${ }^{5}$ Dataset: Microsoft Research Paraphrase Corpus (MRPC) (Dolan and Brockett, 2005).

\section{Evolution of syntax trees}

Hewitt and Manning (2019)'s structural probe evaluates how well syntax trees are embedded in a linear transformation of the network representation space, performing two different evaluations: i) Tree distance evaluation, in which squared L2 distance encodes the distance between words in the parse tree, and ii) Tree depth evaluation, in which squared L2 norm encodes the depth of the parse tree.

Using their probe, Hewitt and Manning show that the 7th layer of BERT-base is the layer that encodes more syntactic information. Therefore, to analyze the evolution of the encoded syntax trees, we train the probes on the 7th layer of the different checkpoint models generated along the fine-tuning process of each task. ${ }^{6}$

\subsection{Tree distance evaluation}

The probe evaluates how well the predicted distances between all pairs of words in a model reconstruct gold parse trees by computing the Undirected Unlabeled Attachment Score (UUAS). It also computes the Spearman correlation between true and predicted distances for each word in each sentence, averaging across all sentences with lengths between 5 and 50 (henceforth referred to as $D S p r$.).

Morpho-syntactic tasks As shown in Figures 1 and 2, both metrics follow a similar behaviour (shades represent the variability across the 5 model runs). PoS tagging shows an important loss of performance all along the fine-tuning process, especially noticeable for UUAS (Figure 1), suggesting that distance-related syntactic information is of less relevance to PoS tagging than could be intuitively

\footnotetext{
${ }^{4}$ Source: https://github.com/huggingface/ transformers/tree/master/examples/ question-answering.

${ }^{5}$ Source: https://github.com/huggingface/ transformers/blob/master/examples/ text-classification/run_glue.py.

${ }^{6} \mathrm{Cf}$. also Supplementary Material.
}

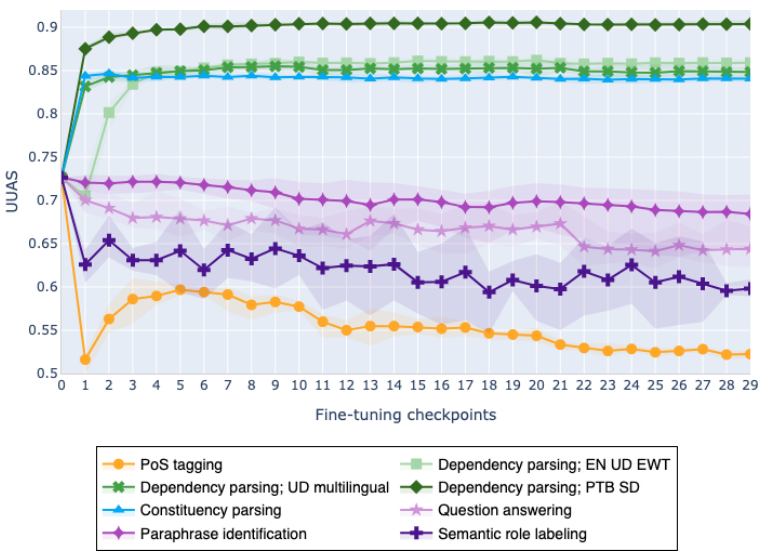

Figure 1: Tree distance evaluation. UUAS evolution.

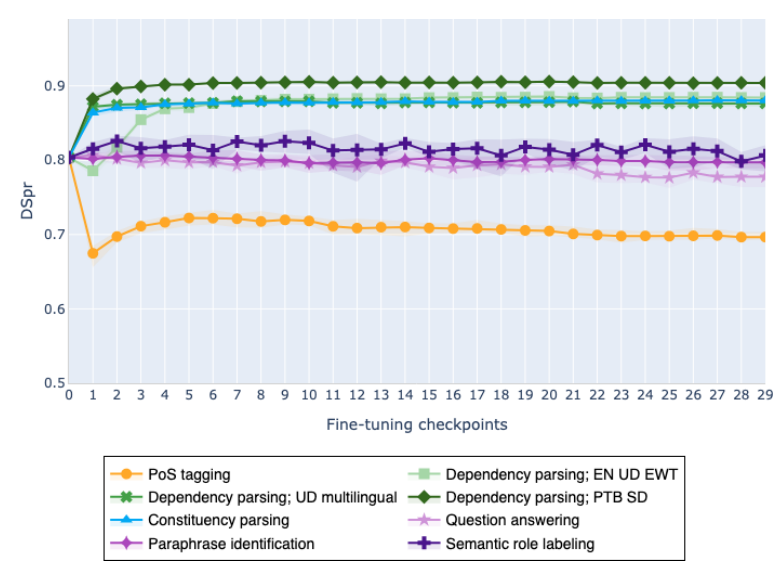

Figure 2: Tree distance evaluation. Dspr evolution.

assumed. As many words have a clear preference towards a specific PoS, especially in English, and most of the ambiguous cases can be resolved using information in the close vicinity (e.g., a simple 3gram sequence tagger is able to achieve a very high accuracy (Manning, 2011)), syntactic structure information may not be necessary and, therefore, the model does not preserve it. This observation is aligned with Pimentel et al. (2020), who found that PoS-tagging is not an ideal task for contemplating the syntax contained in contextual word embeddings. The loss is less pronounced on depth-related metrics, maybe because the root of the sentence usually corresponds to the verb, which may also help in identifying the PoS of surrounding words.

Constituency parsing and dependency parsing share a very similar tendency, with a big improvement in the first fine-tuning steps preserved along the rest of the process. As both tasks heavily rely on syntactic information, this improvement intuitively makes sense. Dependency parsing fine-tuned on the Penn Treebank (PTB) shows even higher results since the probing is trained on the same dataset. In- 


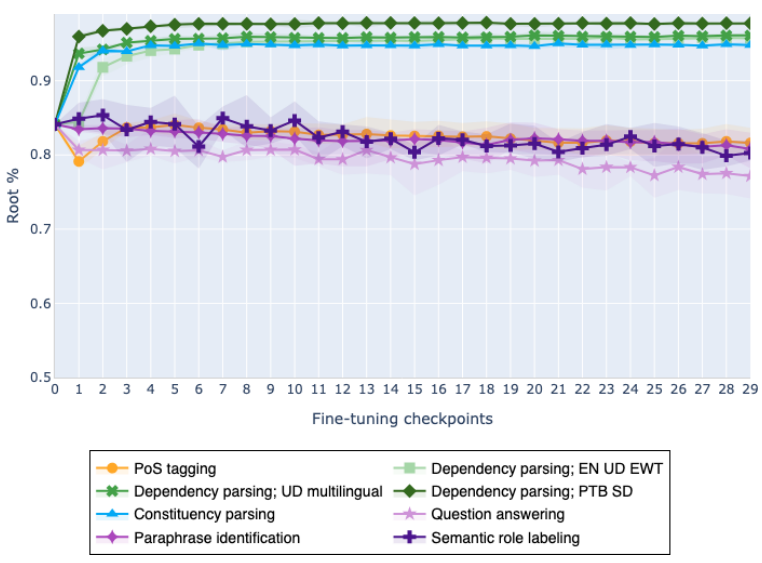

Figure 3: Tree depth evaluation. Root \% evolution.

terestingly, the probe performs similarly even if the parsing task is modeled as a sequence labeling problem (as in constituency parsing), suggesting that the structure of syntax trees emerges in such models even when no tree is explicitly involved in the task. The initial drop observed for PoS tagging and monolingual dependency parsing with UD, trained on UD EN EWT, may be related to the size of the dataset, since UD EN EWT is significantly smaller than the other datasets and therefore the models see less examples per checkpoint.

Semantics-related tasks As shown in Figures 1 and 2, both metrics follow different behaviours (again, shades represent the variability across the 5 model runs). Paraphrase identification shows a small but constant UUAS loss along the fine-tuning, while QA shows a slightly steeper loss trend. Initially, SRL loses around 12 points, suggesting that it discards some syntactic information right at the beginning, and follows a similar downward trend afterwards. Those three tasks show a stable performance along the fine-tuning for the DSpr metric, which implies that even if there is a loss in UUAS information it does not impact the distance ordering.

\subsection{Tree depth evaluation}

The probe evaluates models with respect to their ability to recreate the order of words specified by their depth in the parse tree, assessing their ability to identify the root of the sentence as the least deep word (Root \%) and computing the Spearman correlation between the predicted and the true depth ordering, averaging across all sentences with lengths between 5 and 50 (henceforth referred to as NSpr).

Morpho-syntactic tasks Again, both metrics follow a similar behaviour, as shown in Figures 3 and

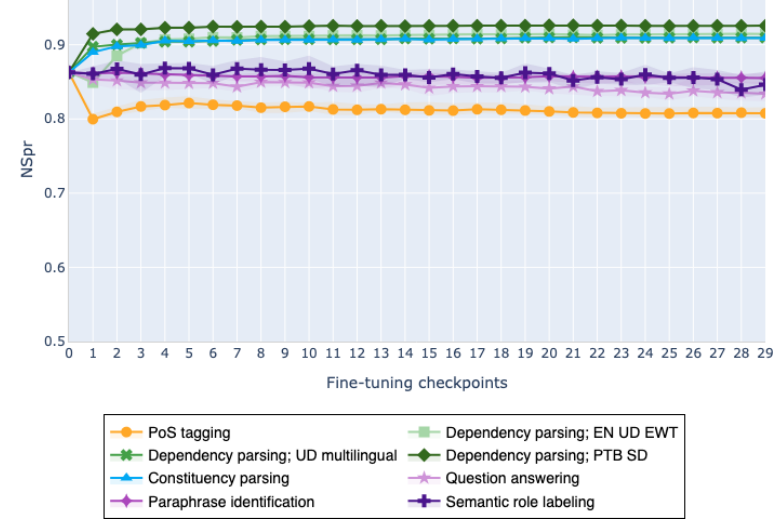

Figure 4: Tree depth evaluation. Nspr evolution.

4. PoS tagging shows a sustained loss of performance, though softer than the loss observed for the distance metrics. This loss is slightly less pronounced for Root \% than for Nspr, suggesting that while depth-related syntactic information may be of less relevance to PoS tagging than it is to the other morpho-syntactic tasks, identifying the root of the sentence may be important, as the root of the sentence is likely to become one of the ambiguous tags and therefore identifying it may help to select the correct label. Constituency parsing and dependency parsing share a similar tendency, with a big improvement in the first steps preserved along the rest of the fine-tuning process, reinforcing the intuition previously introduced in Section 4.1 about the structure of syntax trees emerging in models even when no tree is explicitly involved in the task. Again, an initial drop can be observed for PoS tagging and monolingual dependency parsing with UD, most probably related to the smaller size of the UD EN EWT dataset used in both tasks.

Semantics-related tasks Both metrics follow a similar behaviour, as shown in Figures 3 and 4, with all tasks following a soft but sustained loss of performance until the end of the fine-tuning process, specially noticeable for Root \%.

\section{Conclusions}

We show that fine-tuning is not always a conservative process. Rather, the syntactic information initially encoded in the models is forgotten (PoS tagging), reinforced (parsing) or preserved (semanticsrelated tasks) in different (sometimes unexpected) ways along the fine-tuning, depending on the task. Thus, we expected that morpho-syntactic tasks clearly reinforce syntactic information. However, 
PoS tagging forgets it, which, on the other side, can also be justified linguistically (cf. Section 4.1). In contrast, tasks closer to semantics mostly preserve the syntactic knowledge initially encoded. This interesting observation reinforces recent findings that models benefit from explicitly injecting syntactic information for such tasks (Singh Sachan et al., 2020).

Overall, we observed that morpho-syntactic tasks experiment substantial changes in the initial phases, while semantic-related tasks maintain a more stable trend, highlighting the importance of syntactic information in tasks that are not explicitly syntactic in nature (Kuncoro et al., 2020). These observations lead to some interesting insights, but also to further questions; for instance: Can we find a specific set of probes covering different linguistic phenomena to be used as a pretraining stopping criteria? Would this lead to an improvement in the encoding of the linguistic information on pretrained models?

\section{Acknowledgments}

This work has been partially funded by the European Commission via its H2020 Research Program under the contract numbers 779962, 786731, 825079 , and 870930.

\section{References}

Yossi Adi, Einat Kermany, Yonatan Belinkov, Ofer Lavi, and Yoav Goldberg. 2016. Fine-grained analysis of sentence embeddings using auxiliary prediction tasks. arXiv preprint arXiv:1608.04207.

Yonatan Belinkov, Nadir Durrani, Fahim Dalvi, Hassan Sajjad, and James Glass. 2017. What do neural machine translation models learn about morphology? In Proceedings of the 55th Annual Meeting of the Association for Computational Linguistics (Volume 1: Long Papers), pages 861-872, Vancouver, Canada. Association for Computational Linguistics.

Anders Björkelund, Bernd Bohnet, Love Hafdell, and Pierre Nugues. 2010. A high-performance syntactic and semantic dependency parser. In Proceedings of the 23rd International Conference on Computational Linguistics: Demonstrations, pages 3336. Association for Computational Linguistics.

Xavier Carreras and Lluís Màrquez. 2005. Introduction to the CoNLL-2005 shared task: Semantic role labeling. In Proceedings of the Ninth Conference on Computational Natural Language Learning (CoNLL-2005), pages 152-164, Ann Arbor, Michigan. Association for Computational Linguistics.
Ethan A. Chi, John Hewitt, and Christopher D. Manning. 2020. Finding universal grammatical relations in multilingual bert.

Alexis Conneau, German Kruszewski, Guillaume Lample, Loïc Barrault, and Marco Baroni. 2018. What you can cram into a single $\$ \&$ !\#* vector: Probing sentence embeddings for linguistic properties. In Proceedings of the 56th Annual Meeting of the Association for Computational Linguistics (Volume 1: Long Papers), pages 2126-2136, Melbourne, Australia. Association for Computational Linguistics.

Marie-Catherine De Marneffe, Bill MacCartney, Christopher D Manning, et al. 2006. Generating typed dependency parses from phrase structure parses. In Lrec, volume 6, pages 449-454.

Jacob Devlin, Ming-Wei Chang, Kenton Lee, and Kristina Toutanova. 2019. BERT: Pre-training of deep bidirectional transformers for language understanding. In Proceedings of the 2019 Conference of the North American Chapter of the Association for Computational Linguistics: Human Language Technologies, Volume 1 (Long and Short Papers), pages 4171-4186, Minneapolis, Minnesota. Association for Computational Linguistics.

Jesse Dodge, Gabriel Ilharco, Roy Schwartz, Ali Farhadi, Hannaneh Hajishirzi, and Noah Smith. 2020. Fine-tuning pretrained language models: Weight initializations, data orders, and early stopping. arXiv preprint arXiv:2002.06305.

William B Dolan and Chris Brockett. 2005. Automatically constructing a corpus of sentential paraphrases. In Proceedings of the Third International Workshop on Paraphrasing (IWP2005).

Timothy Dozat and Christopher D. Manning. 2016. Deep biaffine attention for neural dependency parsing.

Nadir Durrani, Hassan Sajjad, Fahim Dalvi, and Yonatan Belinkov. 2020. Analyzing individual neurons in pre-trained language models. In Proceedings of the 2020 Conference on Empirical Methods in Natural Language Processing (EMNLP), pages 4865-4880, Online. Association for Computational Linguistics.

Yanai Elazar, Shauli Ravfogel, Alon Jacovi, and Yoav Goldberg. 2020. When bert forgets how to pos: Amnesic probing of linguistic properties and $\mathrm{mlm}$ predictions.

Allyson Ettinger. 2020. What bert is not: Lessons from a new suite of psycholinguistic diagnostics for language models. Transactions of the Association for Computational Linguistics, 8:34-48.

Matt Gardner, Joel Grus, Mark Neumann, Oyvind Tafjord, Pradeep Dasigi, Nelson F. Liu, Matthew Peters, Michael Schmitz, and Luke S. Zettlemoyer. 2017. Allennlp: A deep semantic natural language processing platform. 
Jon Gauthier and Roger Levy. 2019. Linking artificial and human neural representations of language. In Proceedings of the 2019 Conference on Empirical Methods in Natural Language Processing and the 9th International Joint Conference on Natural Language Processing (EMNLP-IJCNLP), pages 529539, Hong Kong, China. Association for Computational Linguistics.

Yoav Goldberg. 2019. Assessing bert's syntactic abilities. arXiv preprint arXiv:1901.05287.

James Henderson. 2020. The unstoppable rise of computational linguistics in deep learning.

John Hewitt and Christopher D Manning. 2019. A structural probe for finding syntax in word representations. In Proceedings of the 2019 Conference of the North American Chapter of the Association for Computational Linguistics: Human Language Technologies, Volume 1 (Long and Short Papers), pages 4129-4138.

Ganesh Jawahar, Benoît Sagot, and Djamé Seddah. 2019. What does BERT learn about the structure of language? In Proceedings of the 57th Annual Meeting of the Association for Computational Linguistics, pages 3651-3657, Florence, Italy. Association for Computational Linguistics.

Olga Kovaleva, Alexey Romanov, Anna Rogers, and Anna Rumshisky. 2019. Revealing the dark secrets of BERT. In Proceedings of the 2019 Conference on Empirical Methods in Natural Language Processing and the 9th International Joint Conference on Natural Language Processing (EMNLP-IJCNLP), pages 4365-4374, Hong Kong, China. Association for Computational Linguistics.

Artur Kulmizev, Vinit Ravishankar, Mostafa Abdou, and Joakim Nivre. 2020. Do neural language models show preferences for syntactic formalisms? In Proceedings of the 58th Annual Meeting of the Association for Computational Linguistics, pages 40774091, Online. Association for Computational Linguistics.

Adhiguna Kuncoro, Lingpeng Kong, Daniel Fried, Dani Yogatama, Laura Rimell, Chris Dyer, and Phil Blunsom. 2020. Syntactic structure distillation pretraining for bidirectional encoders.

Tomasz Limisiewicz, David Mareček, and Rudolf Rosa. 2020. Universal Dependencies According to BERT: Both More Specific and More General. In Findings of the Association for Computational Linguistics: EMNLP 2020, pages 2710-2722, Online. Association for Computational Linguistics.

Yongjie Lin, Yi Chern Tan, and Robert Frank. 2019. Open sesame: Getting inside bert's linguistic knowledge. Proceedings of the 2019 ACL Workshop BlackboxNLP: Analyzing and Interpreting Neural Networks for NLP.
Tal Linzen and Marco Baroni. 2020. Syntactic structure from deep learning. arXiv preprint arXiv:2004.10827.

Nelson F. Liu, Matt Gardner, Yonatan Belinkov, Matthew E. Peters, and Noah A. Smith. 2019. Linguistic knowledge and transferability of contextual representations. In Proceedings of the 2019 Conference of the North American Chapter of the Association for Computational Linguistics: Human Language Technologies, Volume 1 (Long and Short Papers), pages 1073-1094, Minneapolis, Minnesota. Association for Computational Linguistics.

Christopher D Manning. 2011. Part-of-speech tagging from $97 \%$ to $100 \%$ : is it time for some linguistics? In International conference on intelligent text processing and computational linguistics, pages 171189. Springer.

Mitchell Marcus, Beatrice Santorini, and Mary Ann Marcinkiewicz. 1993. Building a large annotated corpus of english: The penn treebank.

Amil Merchant, Elahe Rahimtoroghi, Ellie Pavlick, and Ian Tenney. 2020. What happens to BERT embeddings during fine-tuning? In Proceedings of the Third BlackboxNLP Workshop on Analyzing and Interpreting Neural Networks for NLP, pages 33-44, Online. Association for Computational Linguistics.

Matthew Peters, Mark Neumann, Mohit Iyyer, Matt Gardner, Christopher Clark, Kenton Lee, and Luke Zettlemoyer. 2018. Deep contextualized word representations. In Proceedings of the 2018 Conference of the North American Chapter of the Association for Computational Linguistics: Human Language Technologies, Volume 1 (Long Papers), pages 2227-2237, New Orleans, Louisiana. Association for Computational Linguistics.

Matthew E. Peters, Sebastian Ruder, and Noah A. Smith. 2019. To tune or not to tune? adapting pretrained representations to diverse tasks. In Proceedings of the 4th Workshop on Representation Learning for NLP (RepL4NLP-2019), pages 7-14, Florence, Italy. Association for Computational Linguistics.

Tiago Pimentel, Josef Valvoda, Rowan Hall Maudslay, Ran Zmigrod, Adina Williams, and Ryan Cotterell. 2020. Information-theoretic probing for linguistic structure. In Proceedings of the 58th Annual Meeting of the Association for Computational Linguistics, pages 4609-4622, Online. Association for Computational Linguistics.

Pranav Rajpurkar, Robin Jia, and Percy Liang. 2018. Know what you don't know: Unanswerable questions for SQuAD. In Proceedings of the 56th Annual Meeting of the Association for Computational Linguistics (Volume 2: Short Papers), pages 784789, Melbourne, Australia. Association for Computational Linguistics. 
Vinit Ravishankar, Artur Kulmizev, Mostafa Abdou, Anders Søgaard, and Joakim Nivre. 2021. Attention can reflect syntactic structure (if you let it). In Proceedings of the 16th Conference of the European Chapter of the Association for Computational Linguistics. Association for Computational Linguistics.

Anna Rogers, Olga Kovaleva, and Anna Rumshisky. 2020. A primer in bertology: What we know about how bert works. arXiv preprint arXiv:2002.12327.

Peng Shi and Jimmy Lin. 2019. Simple bert models for relation extraction and semantic role labeling. arXiv preprint arXiv:1904.05255.

Natalia Silveira, Timothy Dozat, Marie-Catherine De Marneffe, Samuel R Bowman, Miriam Connor, John Bauer, and Christopher D Manning. 2014. A gold standard dependency corpus for english. In LREC, pages 2897-2904.

Devendra Singh Sachan, Yuhao Zhang, Peng Qi, and William Hamilton. 2020. Do syntax trees help pretrained transformers extract information? arXiv $e$ prints, pages arXiv-2008.

Emma Strubell, Patrick Verga, Daniel Andor, David Weiss, and Andrew McCallum. 2018. Linguistically-informed self-attention for semantic role labeling.

Ian Tenney, Dipanjan Das, and Ellie Pavlick. 2019a. BERT rediscovers the classical NLP pipeline. In Proceedings of the 57th Annual Meeting of the Association for Computational Linguistics, pages 45934601, Florence, Italy. Association for Computational Linguistics.

Ian Tenney, Patrick Xia, Berlin Chen, Alex Wang, Adam Poliak, R Thomas McCoy, Najoung Kim, Benjamin Van Durme, Sam Bowman, Dipanjan Das, and Ellie Pavlick. 2019b. What do you learn from context? probing for sentence structure in contextualized word representations. In International Conference on Learning Representations.

David Vilares, Michalina Strzyz, Anders Søgaard, and Carlos Gómez-Rodríguez. 2020. Parsing as pretraining. arXiv preprint arXiv:2002.01685.

Elena Voita and Ivan Titov. 2020. Informationtheoretic probing with minimum description length. In Proceedings of the 2020 Conference on Empirical Methods in Natural Language Processing (EMNLP), pages 183-196, Online. Association for Computational Linguistics.

Alex Wang, Amanpreet Singh, Julian Michael, Felix Hill, Omer Levy, and Samuel Bowman. 2018. GLUE: A multi-task benchmark and analysis platform for natural language understanding. In Proceedings of the 2018 EMNLP Workshop BlackboxNLP: Analyzing and Interpreting Neural Networks for NLP, pages 353-355, Brussels, Belgium. Association for Computational Linguistics.
Yufei Wang, Mark Johnson, Stephen Wan, Yifang Sun, and Wei Wang. 2019. How to best use syntax in semantic role labelling. In Proceedings of the 57th Annual Meeting of the Association for Computational Linguistics, pages 5338-5343, Florence, Italy. Association for Computational Linguistics.

Ralph Weischedel, Martha Palmer, Mitchell Marcus, Eduard Hovy, Sameer Pradhan, Lance Ramshaw, Nianwen Xue, Ann Taylor, Jeff Kaufman, Michelle Franchini, et al. 2013. Ontonotes release 5.0 ldc2013t19. Linguistic Data Consortium, Philadelphia, PA, 23.

Thomas Wolf, Lysandre Debut, Victor Sanh, Julien Chaumond, Clement Delangue, Anthony Moi, Pierric Cistac, Tim Rault, R'emi Louf, Morgan Funtowicz, and Jamie Brew. 2019. Huggingface's transformers: State-of-the-art natural language processing. ArXiv, abs/1910.03771.

Daniel Zeman, Joakim Nivre, Mitchell Abrams, Noëmi Aepli, Željko Agić, Lars Ahrenberg, Gabrielè Aleksandravičiūte, Lene Antonsen, Katya Aplonova, Maria Jesus Aranzabe, Gashaw Arutie, Masayuki Asahara, Luma Ateyah, Mohammed Attia, Aitziber Atutxa, Liesbeth Augustinus, Elena Badmaeva, Miguel Ballesteros, Esha Banerjee, Sebastian Bank, Verginica Barbu Mititelu, Victoria Basmov, Colin Batchelor, John Bauer, Sandra Bellato, Kepa Bengoetxea, Yevgeni Berzak, Irshad Ahmad Bhat, Riyaz Ahmad Bhat, Erica Biagetti, Eckhard Bick, Agnė Bielinskiené, Rogier Blokland, Victoria Bobicev, Loïc Boizou, Emanuel Borges Völker, Carl Börstell, Cristina Bosco, Gosse Bouma, Sam Bowman, Adriane Boyd, Kristina Brokaite, Aljoscha Burchardt, Marie Candito, Bernard Caron, Gauthier Caron, Tatiana Cavalcanti, Gülşen Cebiroğlu Eryiğit, Flavio Massimiliano Cecchini, Giuseppe G. A. Celano, Slavomír Čéplö, Savas Cetin, Fabricio Chalub, Jinho Choi, Yongseok Cho, Jayeol Chun, Alessandra T. Cignarella, Silvie Cinková, Aurélie Collomb, Çağrı Çöltekin, Miriam Connor, Marine Courtin, Elizabeth Davidson, MarieCatherine de Marneffe, Valeria de Paiva, Elvis de Souza, Arantza Diaz de Ilarraza, Carly Dickerson, Bamba Dione, Peter Dirix, Kaja Dobrovoljc, Timothy Dozat, Kira Droganova, Puneet Dwivedi, Hanne Eckhoff, Marhaba Eli, Ali Elkahky, Binyam Ephrem, Olga Erina, Tomaž Erjavec, Aline Etienne, Wograine Evelyn, Richárd Farkas, Hector Fernandez Alcalde, Jennifer Foster, Cláudia Freitas, Kazunori Fujita, Katarína Gajdošová, Daniel Galbraith, Marcos Garcia, Moa Gärdenfors, Sebastian Garza, Kim Gerdes, Filip Ginter, Iakes Goenaga, Koldo Gojenola, Memduh Gökırmak, Yoav Goldberg, Xavier Gómez Guinovart, Berta González Saavedra, Bernadeta Griciūtè, Matias Grioni, Normunds Grūzītis, Bruno Guillaume, Céline Guillot-Barbance, Nizar Habash, Jan Hajič, Jan Hajič jr., Mika Hämäläinen, Linh Hà Mỹ, Na-Rae Han, Kim Harris, Dag Haug, Johannes Heinecke, Felix Hennig, Barbora Hladká, Jaroslava Hlaváčová, Florinel Hociung, Petter Hohle, Jena Hwang, 
Takumi Ikeda, Radu Ion, Elena Irimia, lájídé Ishola, Tomáš Jelínek, Anders Johannsen, Fredrik Jørgensen, Markus Juutinen, Hüner Kaşıkara, Andre Kaasen, Nadezhda Kabaeva, Sylvain Kahane, Hiroshi Kanayama, Jenna Kanerva, Boris Katz, Tolga Kayadelen, Jessica Kenney, Václava Kettnerová, Jesse Kirchner, Elena Klementieva, Arne Köhn, Kamil Kopacewicz, Natalia Kotsyba, Jolanta Kovalevskaite, Simon Krek, Sookyoung Kwak, Veronika Laippala, Lorenzo Lambertino, Lucia Lam, Tatiana Lando, Septina Dian Larasati, Alexei Lavrentiev, John Lee, Phng Lê H’ông, Alessandro Lenci, Saran Lertpradit, Herman Leung, Cheuk Ying Li, Josie Li, Keying Li, KyungTae Lim, Maria Liovina, Yuan Li, Nikola Ljubešić, Olga Loginova, Olga Lyashevskaya, Teresa Lynn, Vivien Macketanz, Aibek Makazhanov, Michael Mandl, Christopher Manning, Ruli Manurung, Cătălina Mărănduc, David Mareček, Katrin Marheinecke, Héctor Martínez Alonso, André Martins, Jan Mašek, Yuji Matsumoto, Ryan McDonald, Sarah McGuinness, Gustavo Mendonça, Niko Miekka, Margarita Misirpashayeva, Anna Missilä, Cătălin Mititelu, Maria Mitrofan, Yusuke Miyao, Simonetta Montemagni, Amir More, Laura Moreno Romero, Keiko Sophie Mori, Tomohiko Morioka, Shinsuke Mori, Shigeki Moro, Bjartur Mortensen, Bohdan Moskalevskyi, Kadri Muischnek, Robert Munro, Yugo Murawaki, Kaili Müürisep, Pinkey Nainwani, Juan Ignacio Navarro Horñiacek, Anna Nedoluzhko, Gunta Nešpore-Bērzkalne, Lng Nguỹên Thị, Huy`ên Nguy ên Thị Minh, Yoshihiro Nikaido, Vitaly Nikolaev, Rattima Nitisaroj, Hanna Nurmi, Stina Ojala, Atul Kr. Ojha, Adédaỵọ Olúòkun, Mai Omura, Petya Osenova, Robert Östling, Lilja Øvrelid, Niko Partanen, Elena Pascual, Marco Passarotti, Agnieszka Patejuk, Guilherme PaulinoPassos, Angelika Peljak-Łapińska, Siyao Peng, Cenel-Augusto Perez, Guy Perrier, Daria Petrova, Slav Petrov, Jason Phelan, Jussi Piitulainen, Tommi A Pirinen, Emily Pitler, Barbara Plank, Thierry Poibeau, Larisa Ponomareva, Martin Popel, Lauma Pretkalniņa, Sophie Prévost, Prokopis Prokopidis, Adam Przepiórkowski, Tiina Puolakainen, Sampo Pyysalo, Peng Qi, Andriela Rääbis, Alexandre Rademaker, Loganathan Ramasamy, Taraka Rama, Carlos Ramisch, Vinit Ravishankar, Livy Real, Siva Reddy, Georg Rehm, Ivan Riabov, Michael Rießler, Erika Rimkute, Larissa Rinaldi, Laura Rituma, Luisa Rocha, Mykhailo Romanenko, Rudolf Rosa, Davide Rovati, Valentin Roșca, Olga Rudina, Jack Rueter, Shoval Sadde, Benoît Sagot, Shadi Saleh, Alessio Salomoni, Tanja Samardžić, Stephanie Samson, Manuela Sanguinetti, Dage Särg, Baiba Saulīe, Yanin Sawanakunanon, Nathan Schneider, Sebastian Schuster, Djamé Seddah, Wolfgang Seeker, Mojgan Seraji, Mo Shen, Atsuko Shimada, Hiroyuki Shirasu, Muh Shohibussirri, Dmitry Sichinava, Aline Silveira, Natalia Silveira, Maria Simi, Radu Simionescu, Katalin Simkó, Mária Šimková, Kiril Simov, Aaron Smith, Isabela Soares-Bastos, Carolyn Spadine, Antonio Stella, Milan Straka, Jana Strnadová, Alane Suhr, Umut
Sulubacak, Shingo Suzuki, Zsolt Szántó, Dima Taji, Yuta Takahashi, Fabio Tamburini, Takaaki Tanaka, Isabelle Tellier, Guillaume Thomas, Liisi Torga, Trond Trosterud, Anna Trukhina, Reut Tsarfaty, Francis Tyers, Sumire Uematsu, Zdeňka Urešová, Larraitz Uria, Hans Uszkoreit, Andrius Utka, Sowmya Vajjala, Daniel van Niekerk, Gertjan van Noord, Viktor Varga, Eric Villemonte de la Clergerie, Veronika Vincze, Lars Wallin, Abigail Walsh, Jing Xian Wang, Jonathan North Washington, Maximilan Wendt, Seyi Williams, Mats Wirén, Christian Wittern, Tsegay Woldemariam, Tak-sum Wong, Alina Wróblewska, Mary Yako, Naoki Yamazaki, Chunxiao Yan, Koichi Yasuoka, Marat M. Yavrumyan, Zhuoran Yu, Zdeněk Žabokrtský, Amir Zeldes, Manying Zhang, and Hanzhi Zhu. 2019. Universal dependencies 2.5. LINDAT/CLARIAH$\mathrm{CZ}$ digital library at the Institute of Formal and Applied Linguistics (ÚFAL), Faculty of Mathematics and Physics, Charles University.

\section{A Target tasks performance evolution}

To complement the results shown in the main paper, we include here the performance curves of the target tasks for which the models are fine-tuned, along with the performance curves of the structural probes metrics, facilitating the comparison of the evolution of the encoded syntax trees information and the target tasks performances.

Along with the performance curves of the four structural probes metrics (UUAS, Nspr, Root \% and $D s p r$ ), the following figures include the performance curves of the target tasks and a brief discussion of the results, to help interpretation. Figure 5 shows the accuracy evolution of PoS tagging. Figures 6, 7 and 8 show the Labeled Attachment Score (LAS) of Dependency parsing with PTB SD, EN UD EWT and UD multilingual, respectively. Figure 9 shows the accuracy evolution of Constituency parsing. Figure 10 shows the F1 score evolution of Question Answering. Figure 11 shows the F1 score and accuracy evolution of Paraphrase identification. Finally, Figure 12 shows the F1 score evolution of Semantic Role Labeling. 
PoS tagging reaches a 0.95 accuracy in only two checkpoints, ending up with a 0.97 on the last checkpoint (Figure 5a). It shows a loss of accuracy for the four probing metrics all along the fine-tuning process, especially noticeable for UUAS (Figure 5b) and Root \% (Figure 5d), suggesting that syntactic information is of less relevance to PoS tagging than could be intuitively assumed. The loss is less pronounced on depth-related metrics, maybe due to the fact that the root of the sentence usually corresponds to the verb, which may also help in identifying the PoS of surrounding words.

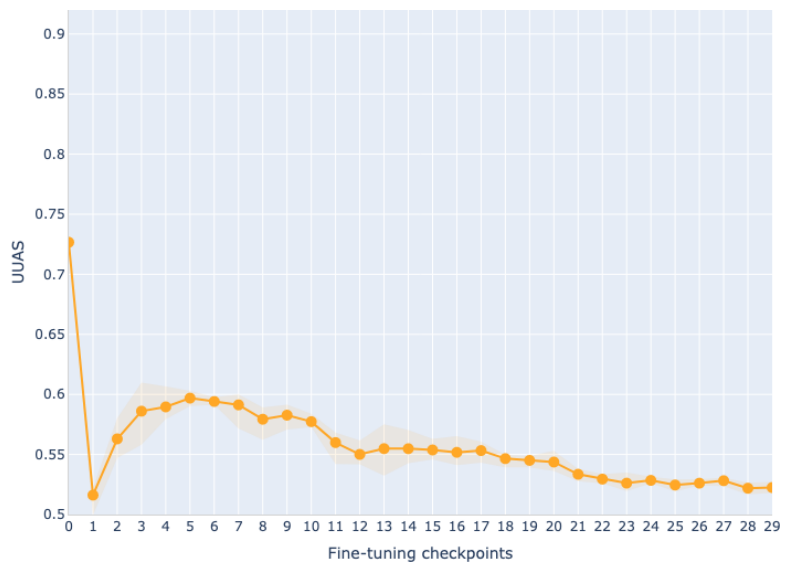

(b) Structural probes tree distance evaluation. UUAS

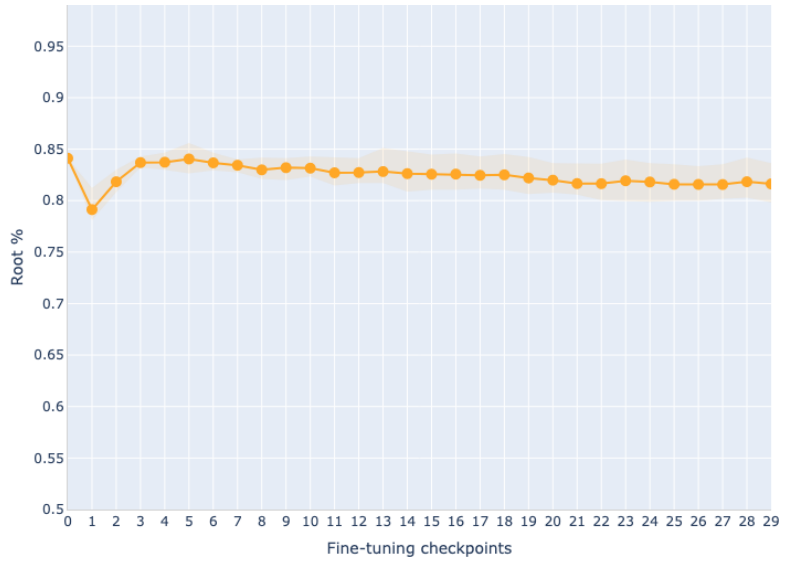

(d) Structural probes tree depth evaluation. Root $\%$

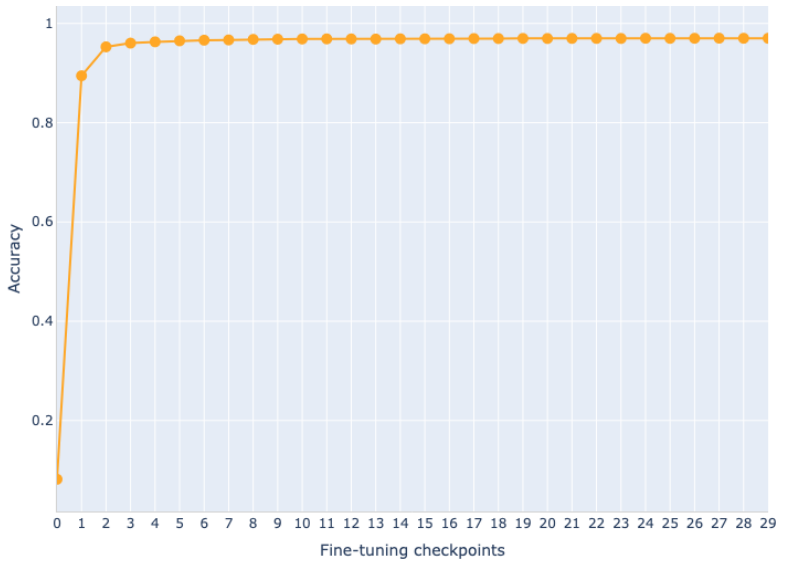

(a) Fine-tuning. Accuracy

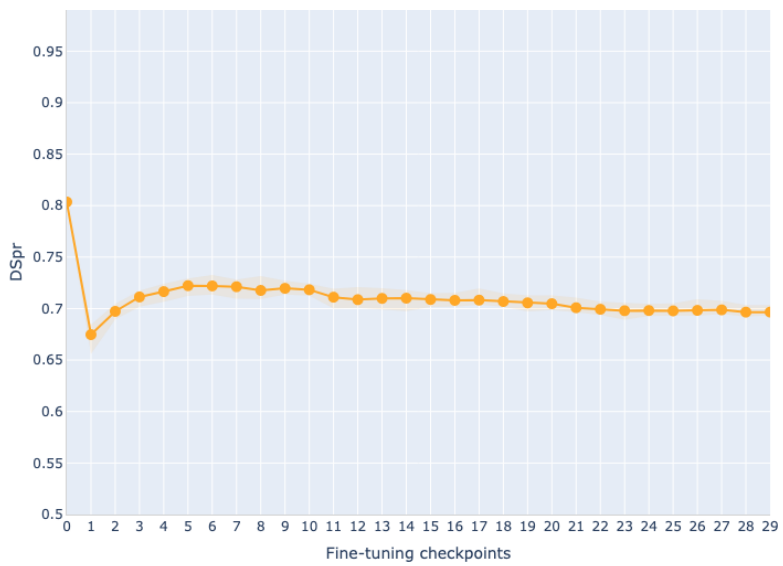

(c) Structural probes tree distance evaluation. Dspr

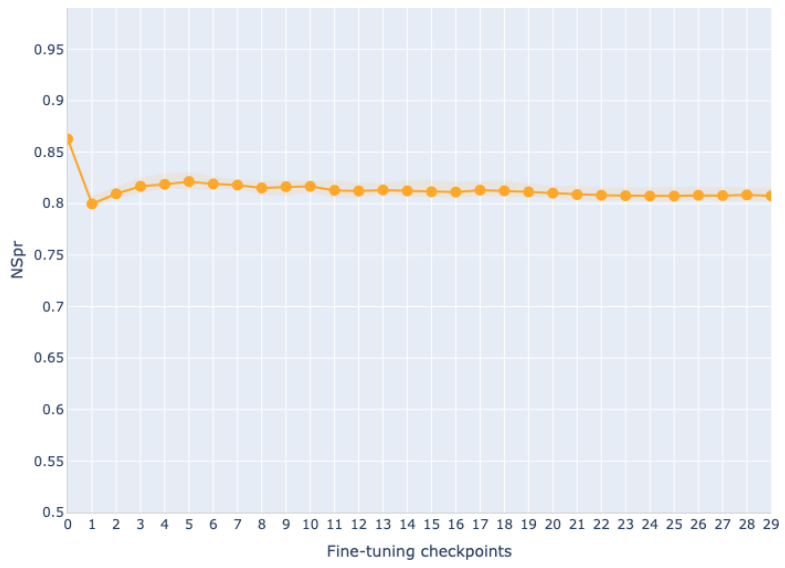

(e) Structural probes tree depth evaluation. Nspr.

Figure 5: POS Tagging. Fine-tuning \& probing metrics evolution. 
Dependency parsing with PTB SD shows a steep learning curve (Figure 6a), reaching a performance of 0.90 LAS on the third checkpoint, up to a final 0.94. All four probing metrics show an important improvement in the first fine-tuning step (Figures 6b, 6c, 6d and 6e), which is preserved along the rest of the process. As the task heavily relies on syntactic information, this improvement intuitively makes sense. Compared to the result of the other dependency parsing experiments, this one show bigger improvements because the probing is trained on the same dataset.

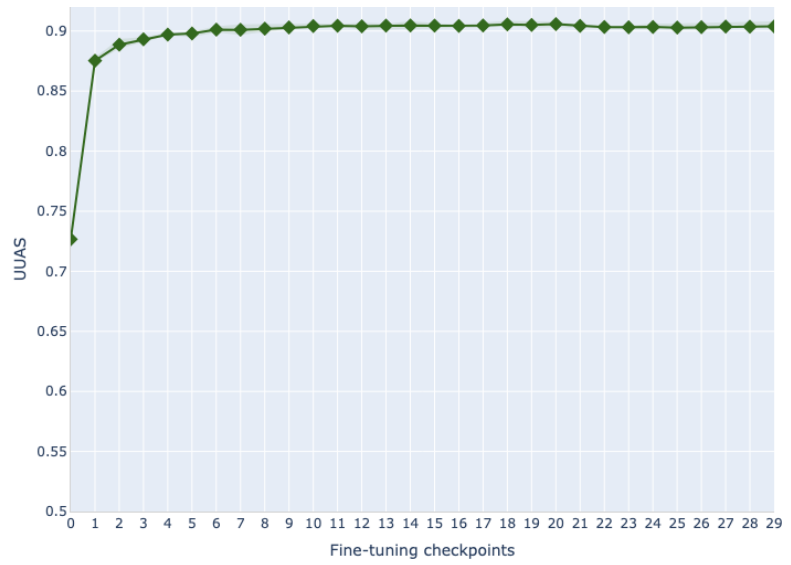

(b) Structural probes tree distance evaluation. UUAS

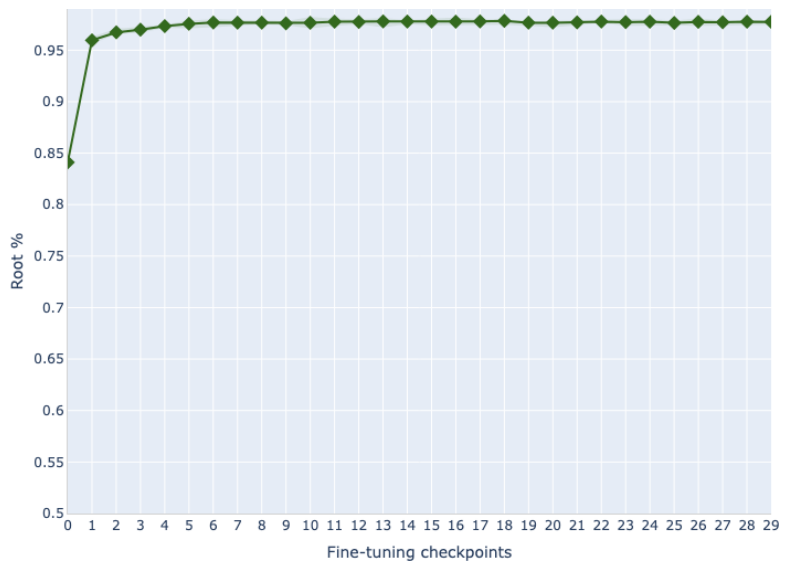

(d) Structural probes tree depth evaluation. Root $\%$

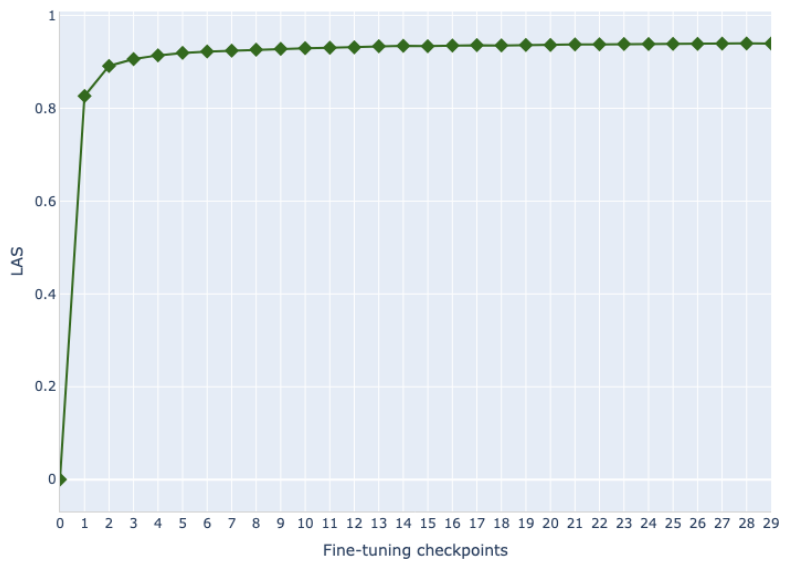

(a) Fine-tuning. LAS

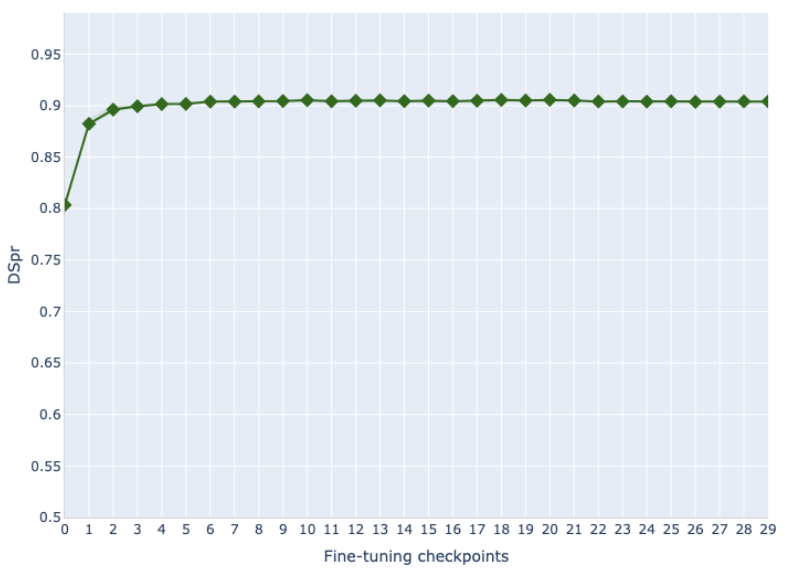

(c) Structural probes tree distance evaluation. Dspr.

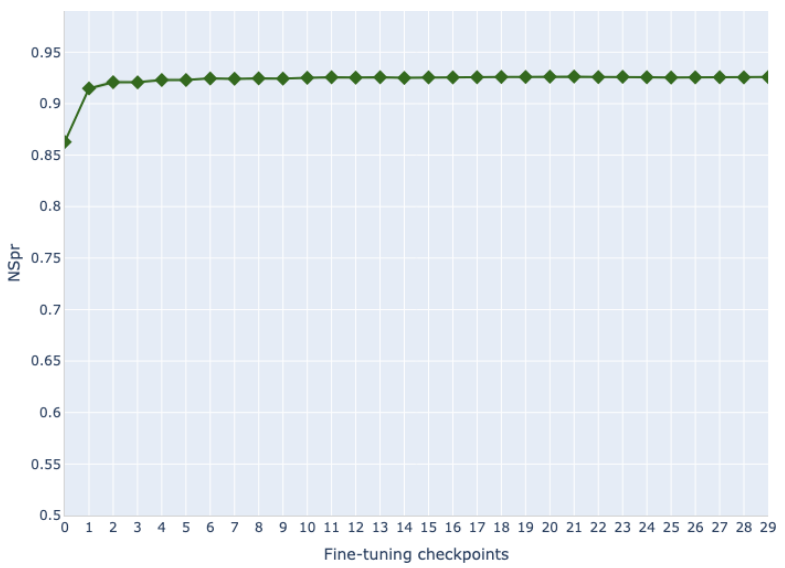

(e) Structural probes tree depth evaluation. Nspr

Figure 6: Dependency Parsing PTB SD. Fine-tuning \& probing metrics evolution. 
Dependency parsing with EN UD EWT shows a shallower learning curve than other experiments (Figure 7a), as the dataset is significantly smaller than the multilingual and PTB and therefore the models see less examples per checkpoint, ending up with a high performance of 0.9 . After an initial drop (probably due to the dataset size, as mentioned before), the probing metrics show a big improvement in the first fine-tuning steps, preserved along the rest of the process (Figures 7b, 7c, 7d and 7e). As the task heavily relies on syntactic information, this improvement intuitively makes sense.

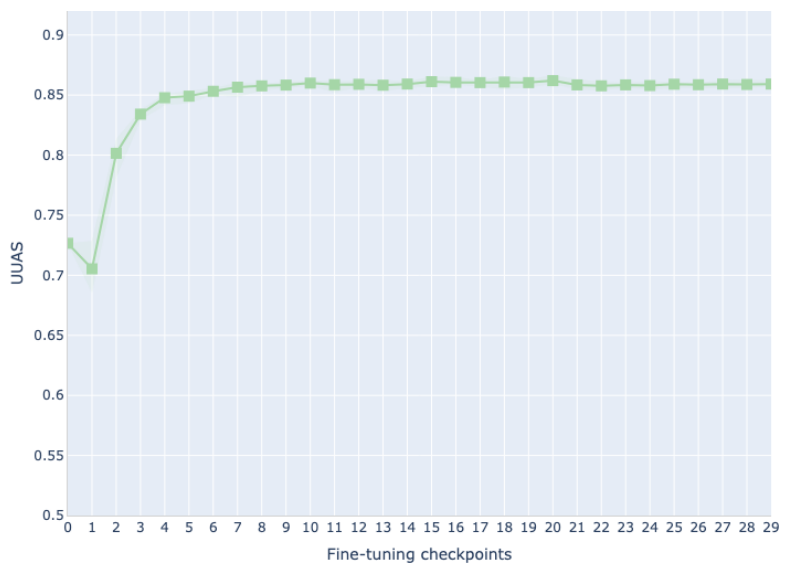

(b) Structural probes tree distance evaluation. UUAS

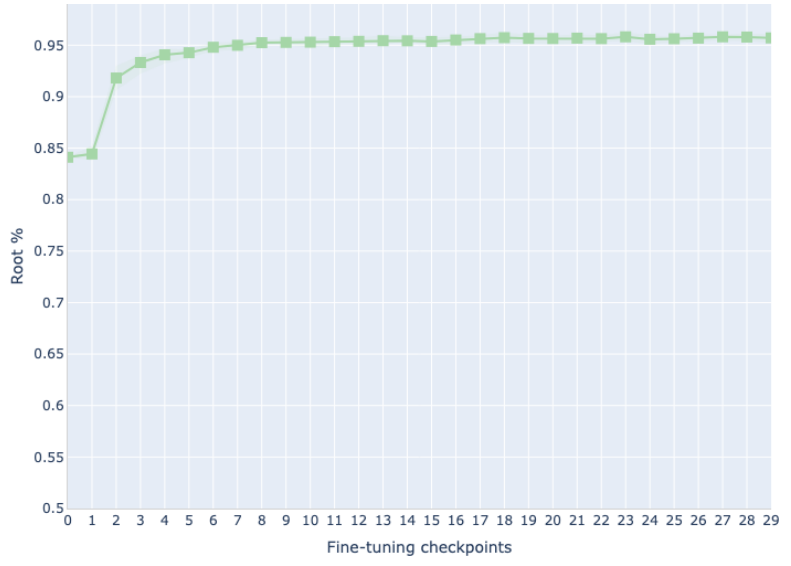

(d) Structural probes tree depth evaluation. Root $\%$

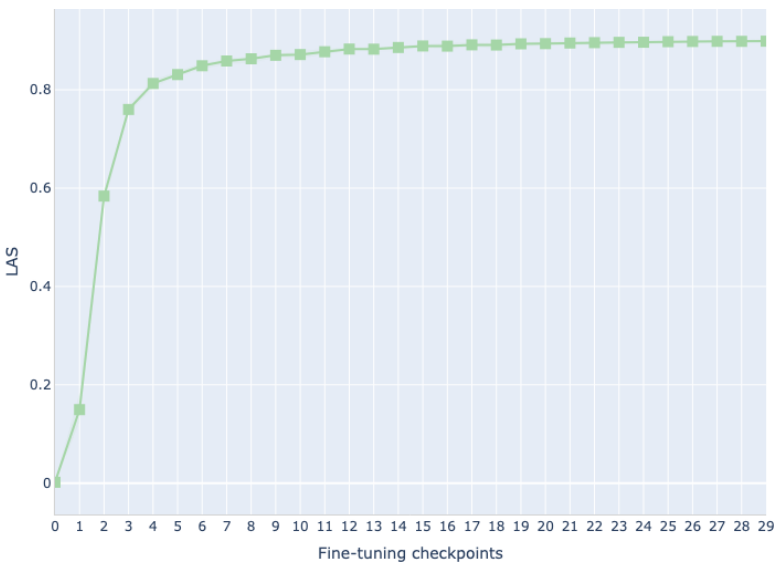

(a) Fine-tuning. LAS

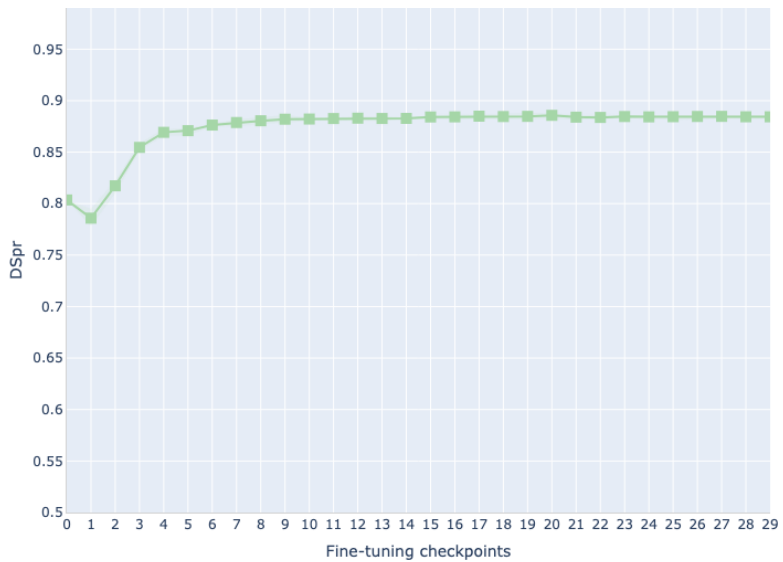

(c) Structural probes tree distance evaluation. Dspr.

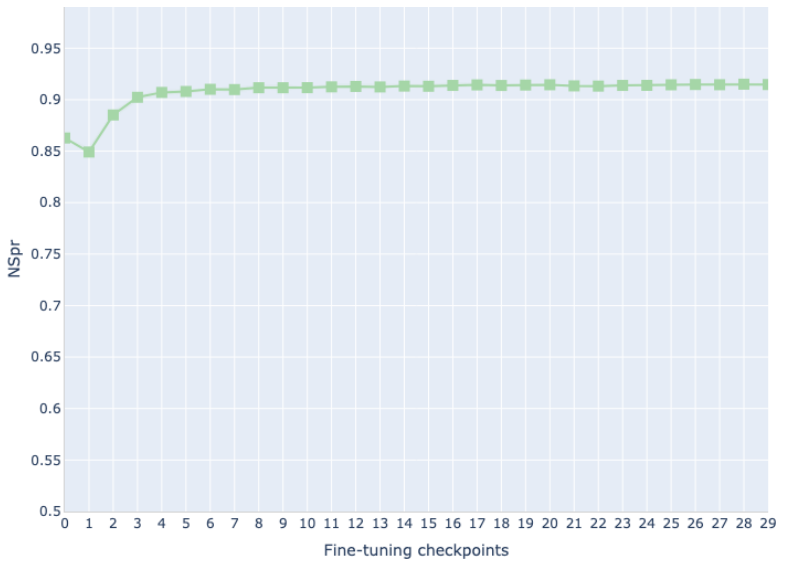

(e) Structural probes tree depth evaluation. Nspr

Figure 7: Dependency Parsing EN UD EWT. Fine-tuning \& probing metrics evolution. 
Multilingual dependency parsing shows a steeper learning curve than dependency parsing with EN UD EWT, as it is trained with a larger dataset (Figure 8a), reaching a performance of 0.87 in LAS. All four probing metrics show a big improvement in the first fine-tuning step, preserved along the rest of the process (Figures 8b, 8c, 8d and $8 \mathrm{e}$ ). As the task heavily relies on syntactic information, this improvement intuitively makes sense.

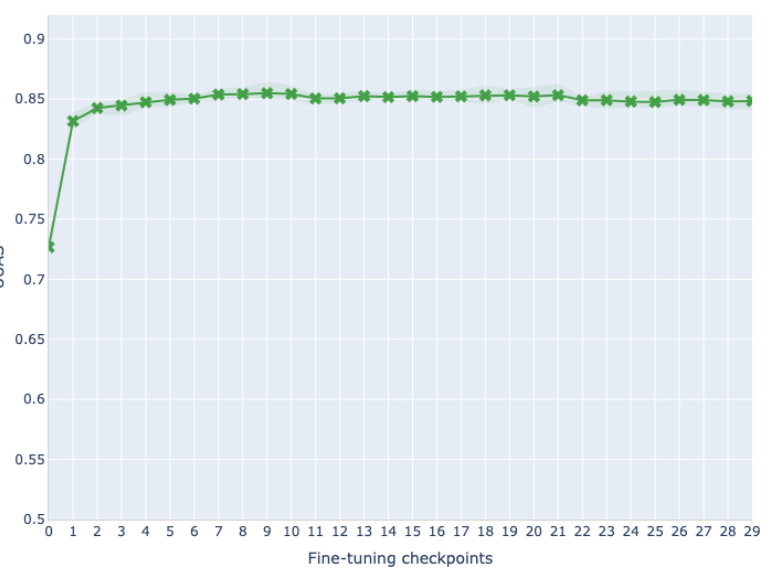

(b) Structural probes tree distance evaluation. UUAS

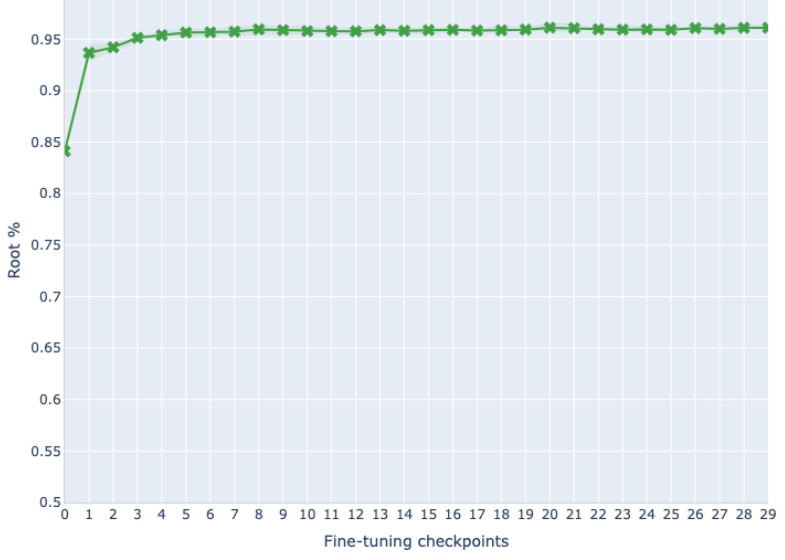

(d) Structural probes tree depth evaluation. Root $\%$

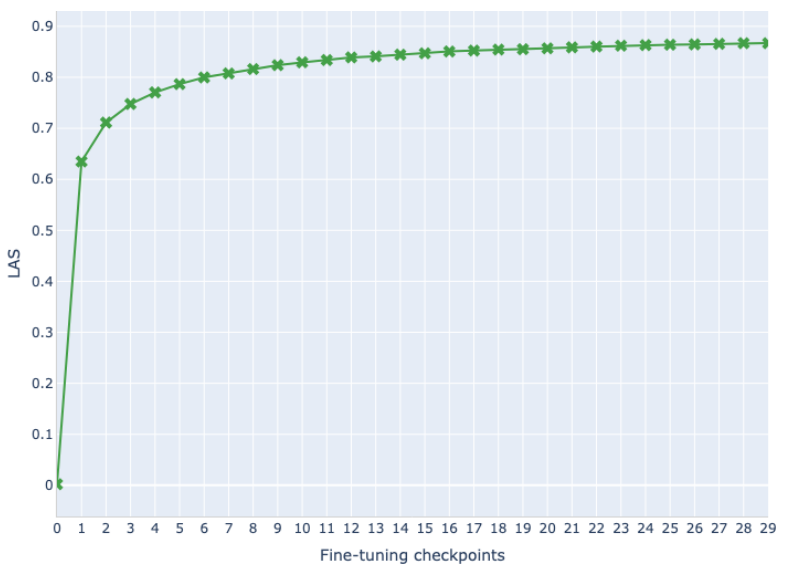

(a) Fine-tuning. LAS

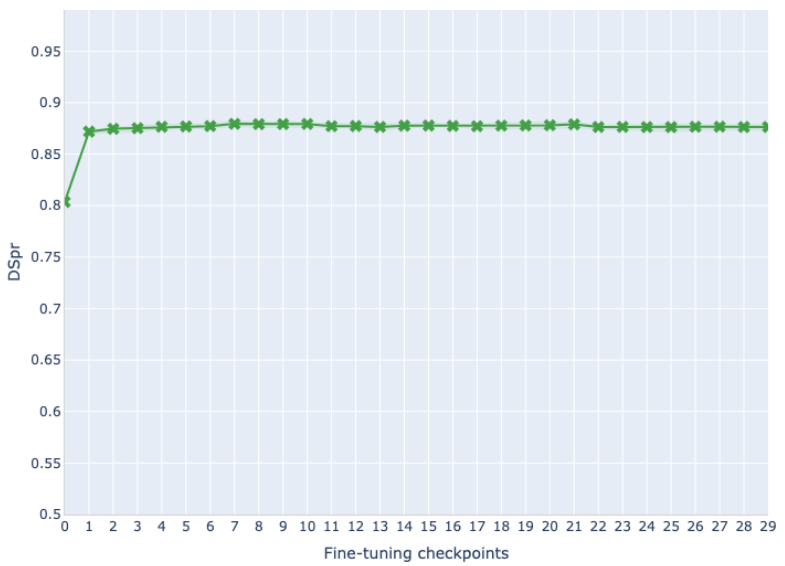

(c) Structural probes tree distance evaluation. Dspr.

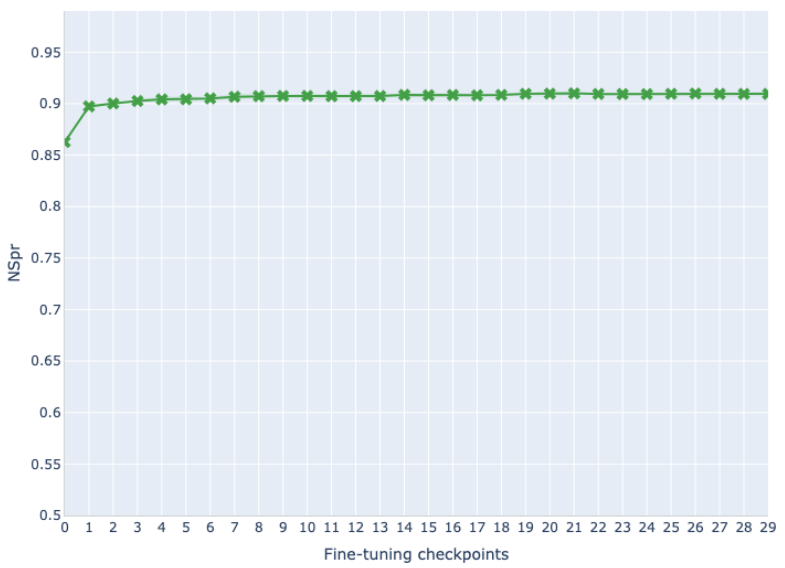

(e) Structural probes tree depth evaluation. Nspr

Figure 8: Dependency Parsing UD Multilingual. Fine-tuning \& probing metrics evolution. 
Constituency parsing fine-tuning follows a steep curve, quickly reaching an Accuracy of 0.87 that is further improved to 0.9 in the last checkpoint (Figure 9a). All four probing metrics show a big improvement in the first fine-tuning steps, preserved along the rest of the process (Figures 9b, 9c, 9d and $9 e)$. As the task heavily relies on syntactic information, this improvement intuitively makes sense. Interestingly, even though the task is modeled as a sequence labeling problem, the probe performs similarly to the dependency parsing tasks, suggesting that the structure of syntax trees emerges in such models even when no tree is explicitly involved in the task.

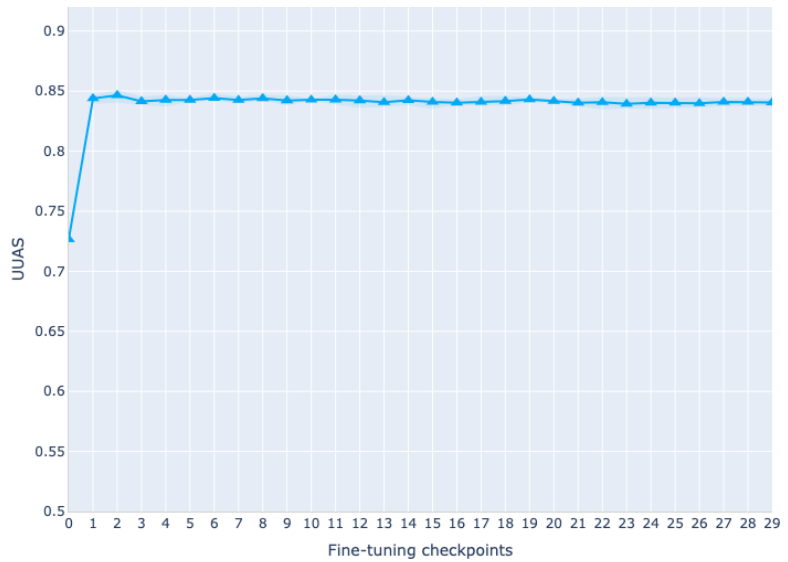

(b) Structural probes tree distance evaluation. UUAS

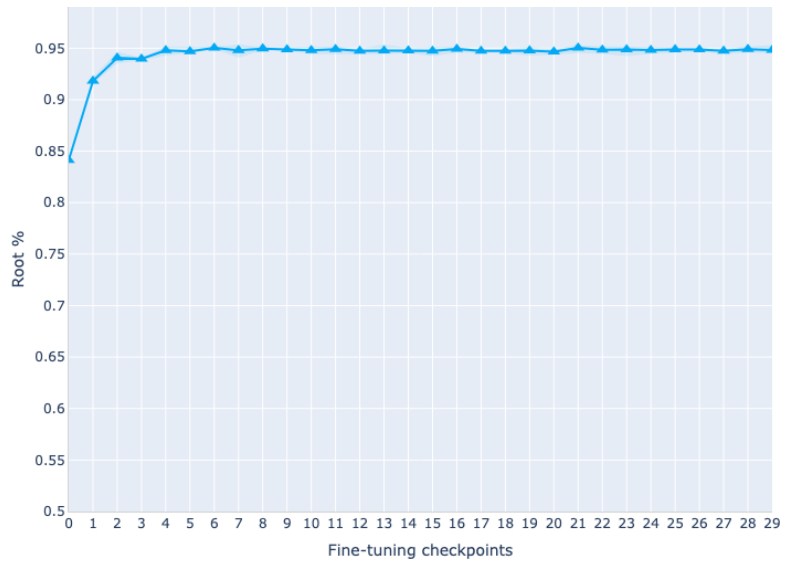

(d) Structural probes tree depth evaluation. Root \%

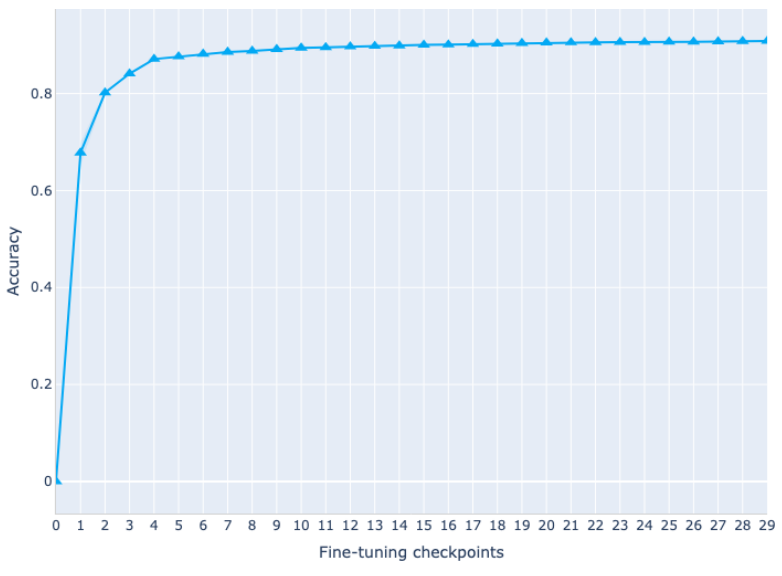

(a) Fine-tuning. Accuracy

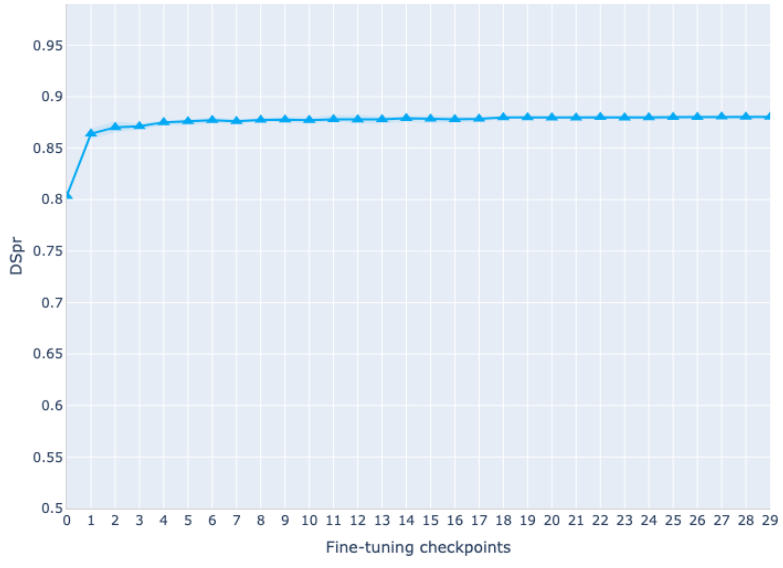

(c) Structural probes tree distance evaluation. Dspr.

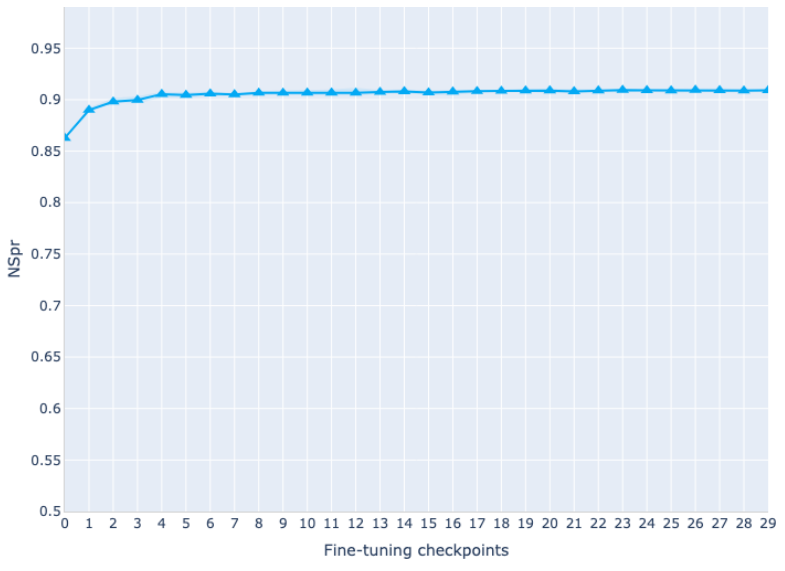

(e) Structural probes tree depth evaluation. Nspr

Figure 9: Constituent Parsing. Fine-tuning \& probing metrics evolution. 
Question answering fine-tuning quickly reaches an F1 score of 0.73 on the first step, which is further improved to 0.88 in the last checkpoint (Figure 10a). All four probing metrics show a clear loss trend (Figures $10 \mathrm{~b}, 10 \mathrm{c}, 10 \mathrm{~d}$ and $10 \mathrm{e}$ ). The loss is specially noticeable for UUAS and Root \%, and more stable for the Spearman correlations, suggesting that even if there is a loss of information it does not impact the distance and depth orderings.

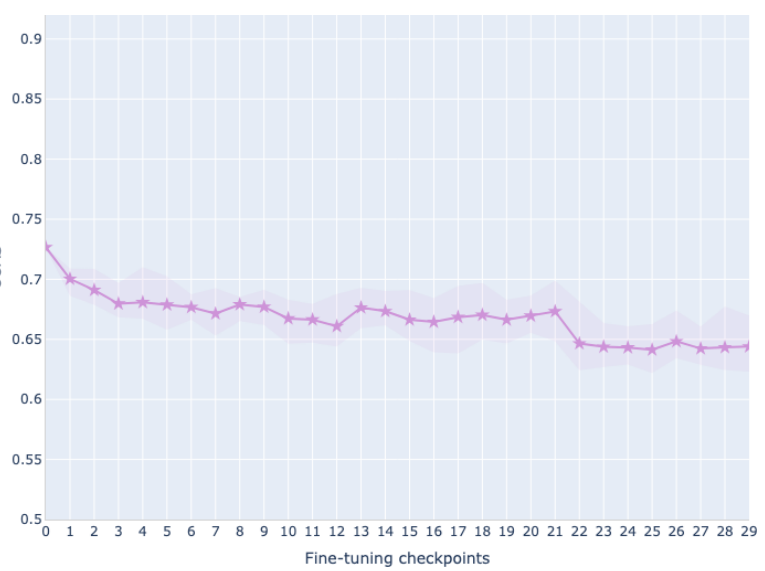

(b) Structural probes tree distance evaluation. UUAS

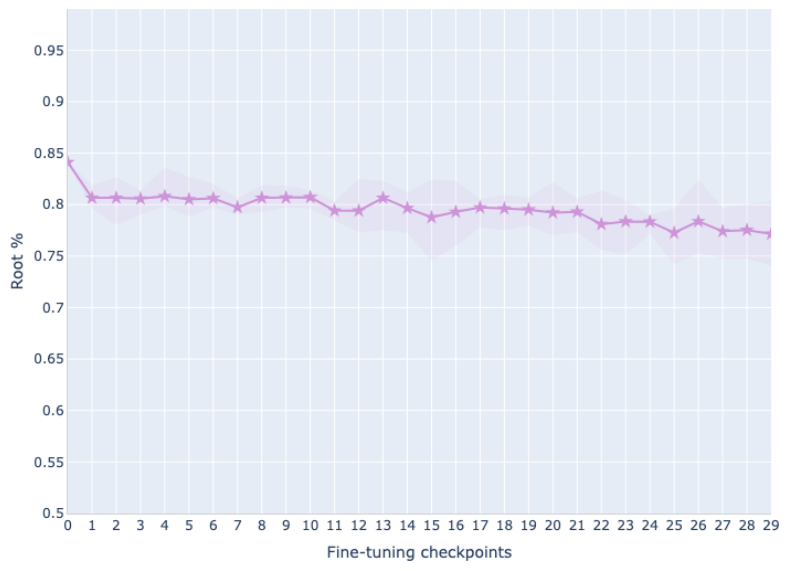

(d) Structural probes tree depth evaluation. Root $\%$

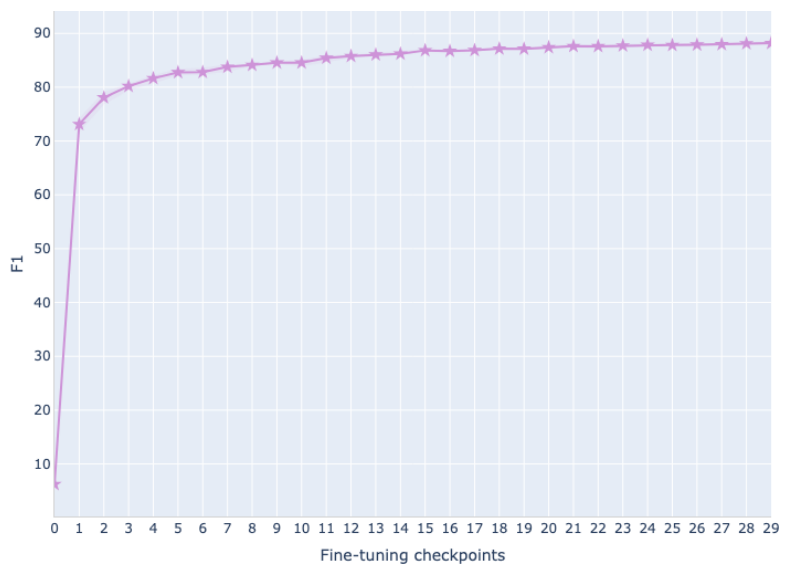

(a) Fine-tuning. F1

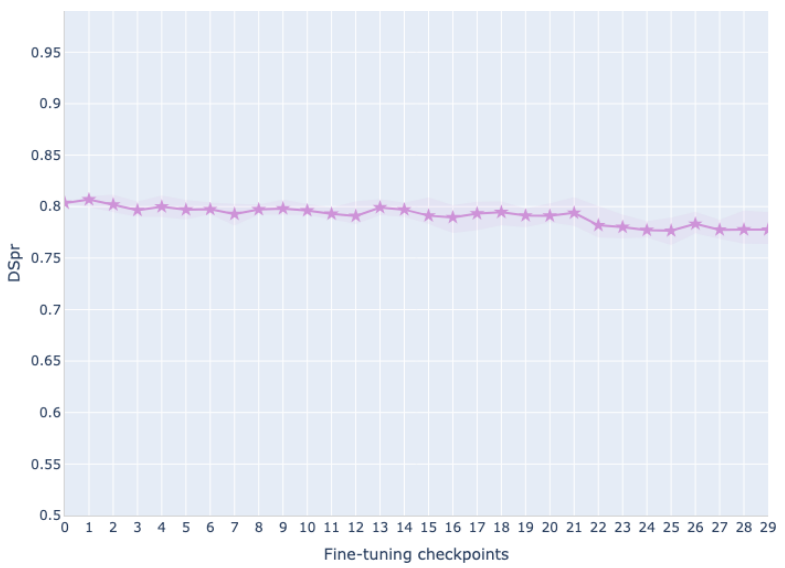

(c) Structural probes tree distance evaluation. Dspr.

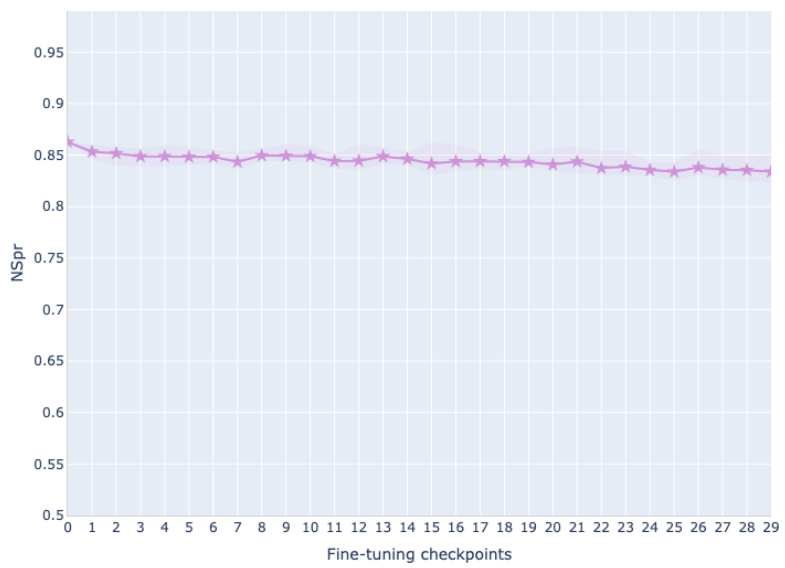

(e) Structural probes tree depth evaluation. Nspr

Figure 10: Question Answering. Fine-tuning \& probing metrics evolution. 
Paraphrase identification fine-tuning starts with an F1 score of 0.81 on the first step that is further improved to 0.90 in the last checkpoint (Figure 11a). Regarding accuracy, after reaching 0.69 on the first checkpoint it follows a shallower curve to a final 0.86 (Figure 11b). All four probing metrics follow a loss trend (Figures 11c, 11d, 11e and 11f). The loss is specially noticeable for UUAS and Root \%, and more stable for the Spearman correlations, suggesting that even if there is a loss of information it does not impact the distance and depth orderings.

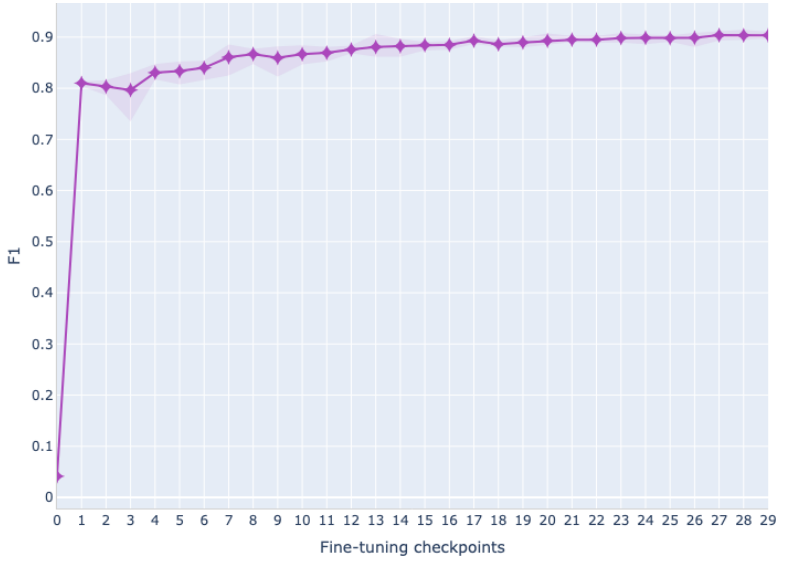

(a) Fine-tuning. F1

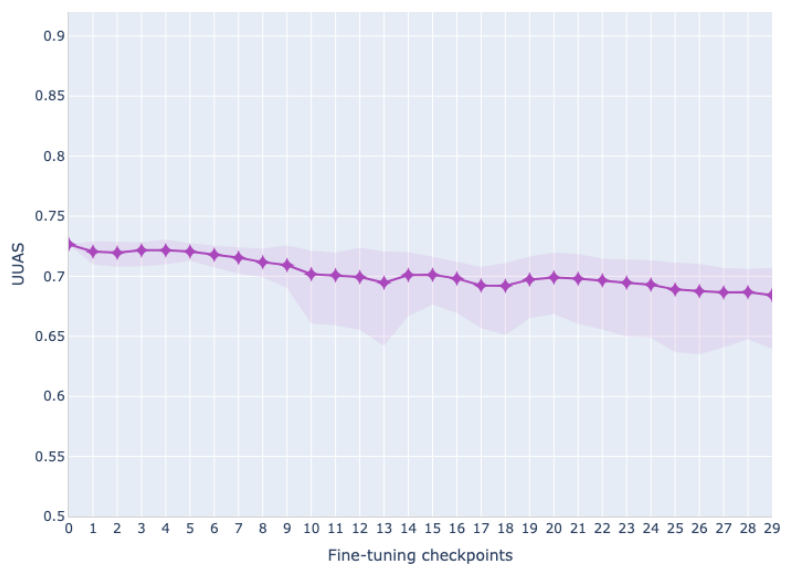

(c) Structural probes tree distance evaluation. UUAS

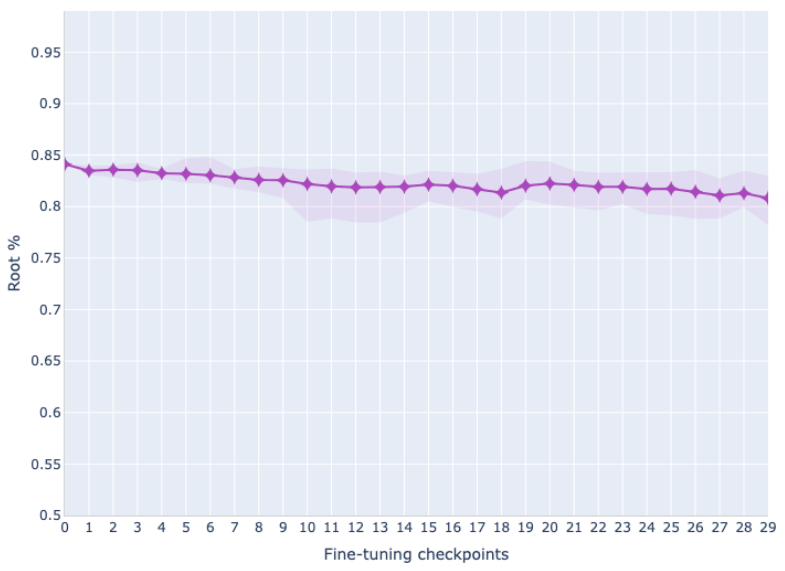

(e) Structural probes tree depth evaluation. Root \%

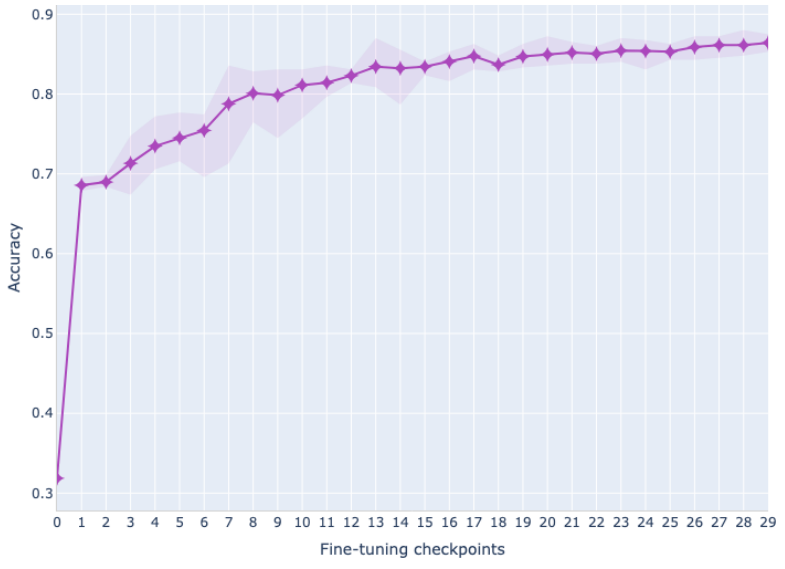

(b) Accuracy

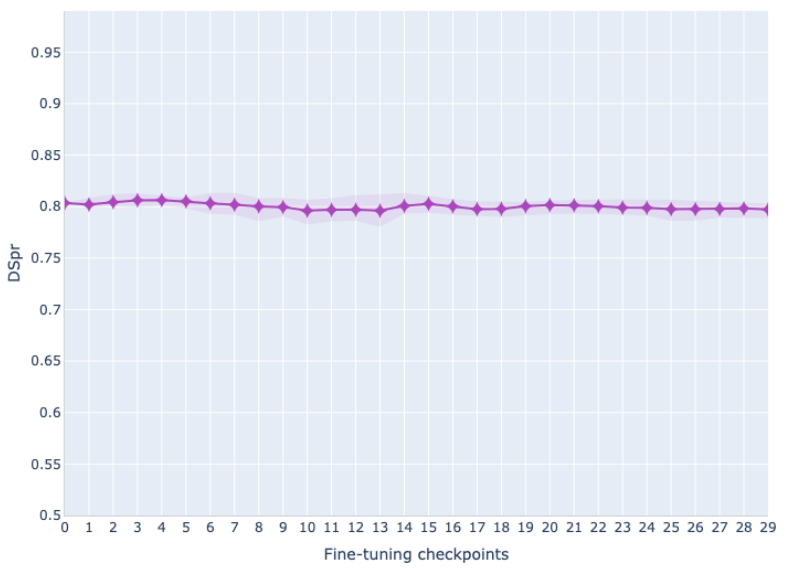

(d) Structural probes tree distance evaluation. Dspr.

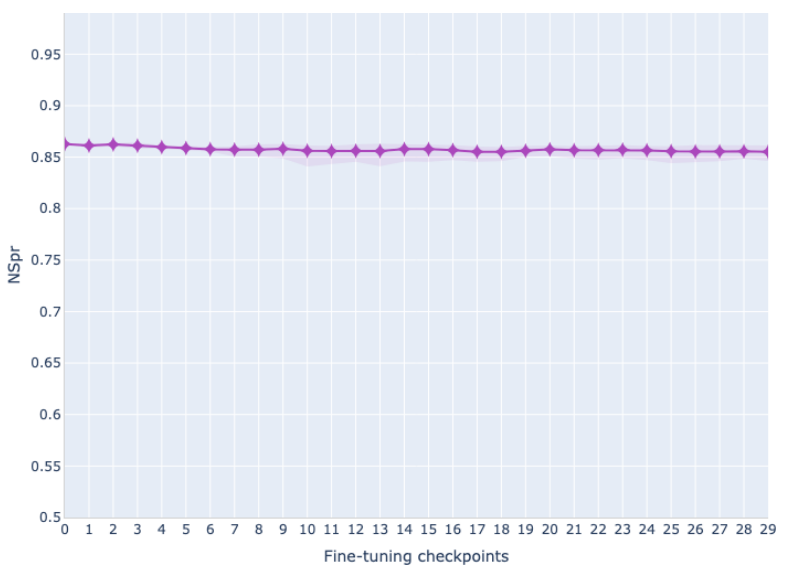

(f) Structural probes tree depth evaluation. Nspr

Figure 11: Paraphrase identification. Fine-tuning \& probing metrics evolution. 
Semantic Role Labeling fine-tuning follows a steep curve for F1, quickly reaching an F1 score of 0.71 on the first step that is further improved to 0.82 in the last checkpoint (Figure 12a). All four probing metrics follow a loss trend (Figures 12b, 12c, 12d and 12e). The loss is specially noticeable for UUAS, which initially loses around 12 UUAS points, and more stable for the Spearman correlations, suggesting that even if there is a loss of information it does not impact the distance and depth orderings.

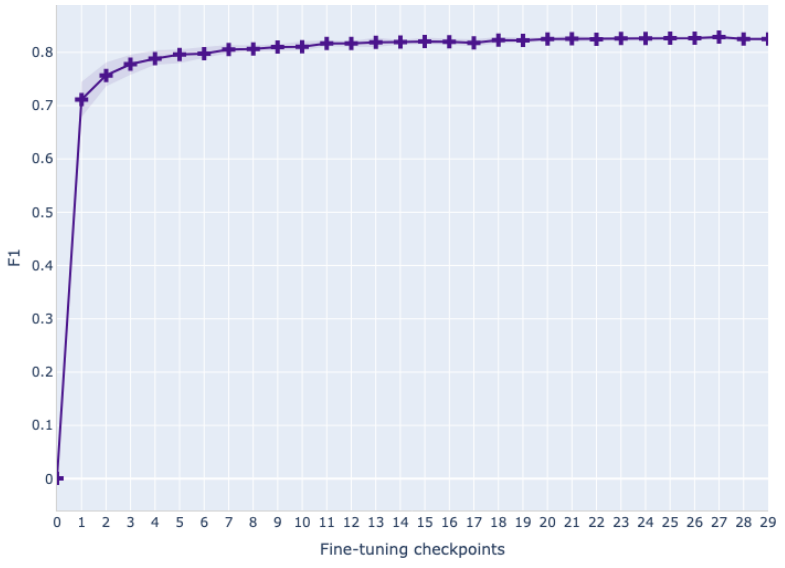

(a) Fine-tuning. F1

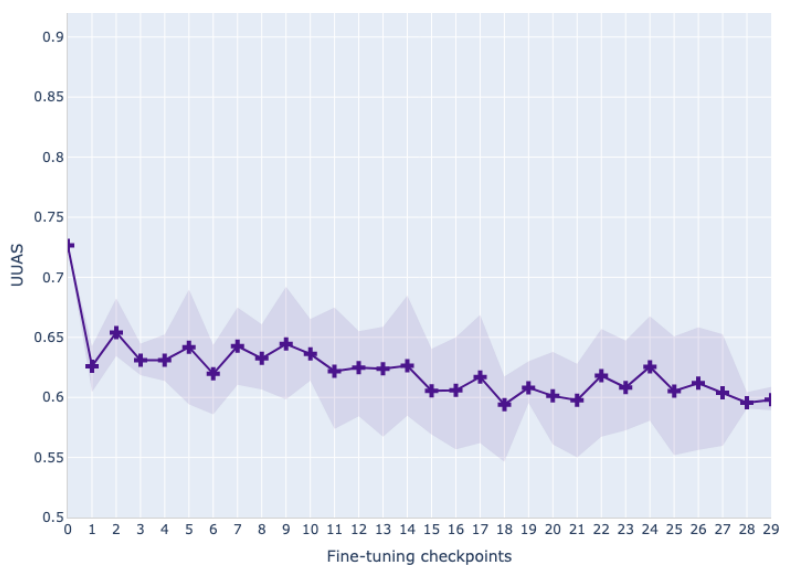

(b) Structural probes tree distance evaluation. UUAS

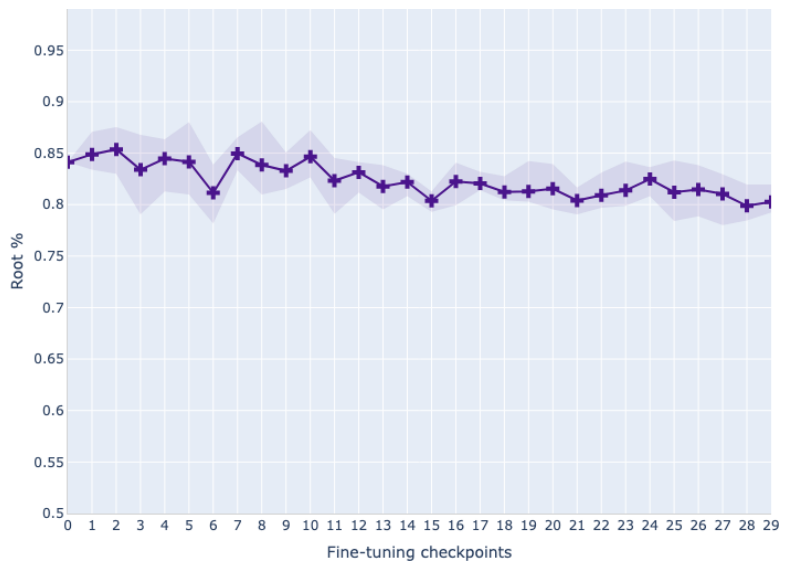

(d) Structural probes tree depth evaluation. Root $\%$

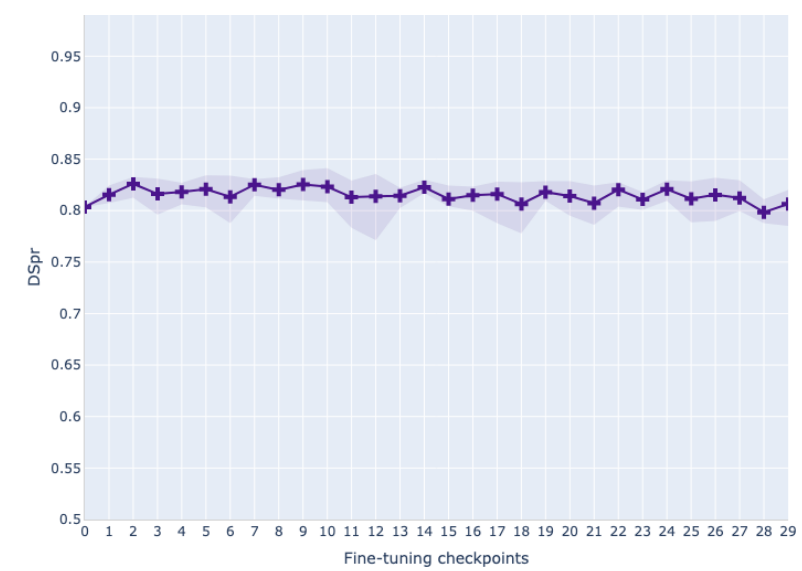

(c) Structural probes tree distance evaluation. Dspr.

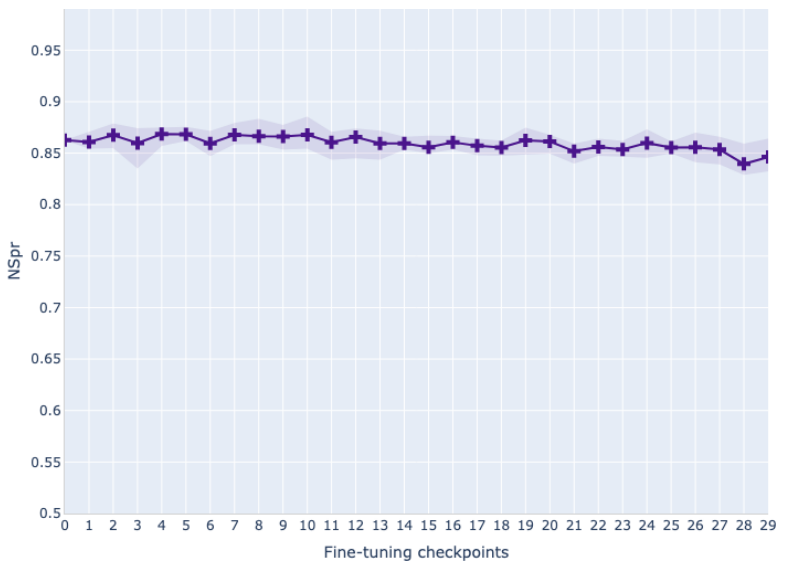

(e) Structural probes tree depth evaluation. Nspr

Figure 12: Semantic Role Labeling. Fine-tuning \& probing metrics evolution. 